\title{
Transceiver Design of Optimum Wirelessly Powered Full-Duplex MIMO IoT Devices
}

\author{
J. Xue, Member, IEEE, S. Biswas, Member, IEEE, \\ A. C. Cirik, Member, IEEE, H. Du, Member, IEEE, Y. Yang, Member, IEEE, \\ T. Ratnarajah, Senior Member, IEEE and M. Sellathurai, Senior Member, IEEE
}

\begin{abstract}
In this paper, we investigate the energy harvesting (EH) technique and accordingly design transceivers for a $K$ link multiple-input multiple-output (MIMO) interference channel. Each link consists of two full-duplex (FD) internet of things (IoT) nodes exchanging information simultaneously in a bi-directional communication channel. All the nodes suffer from interference, in particular strong self-interference and inter-node interference, due to operating in FD mode and simultaneous transmission at each link, respectively. Further, we divide the received signal at each node into two parts. While one part of the signal is used for information decoding, the other part is used for EH. We jointly design the transmit and receive beamforming vectors and receiver power splitting ratios by minimizing the total transmission power of the system, subject to both signal-to-interference-plus-noise ratio (SINR) and EH threshold constraints. Furthermore, the case of multiple-input single-output (MISO) interference channel is also included for the sake of comparison. We also revisit the above problems for the case when the available channel state information (CSI) at the transmitters is imperfect, where the errors of the CSI are assumed to be norm bounded. Simulation results show that the EH technique can harvest enough energy to support power consumption limited IoT devices by aiding in recharging their respective batteries.
\end{abstract}

Index Terms-Beamforming, energy harvesting, full-duplex, MIMO interference channels, power splitting, self-interference.

J. Xue is currently with the National Engineering Laboratory for Big Data Analytics, Xi'an International Academy for Mathematics and Mathematical Technology, School of Mathematics and Statistics, Xi'an Jiaotong University, Xi' an, Shaanxi, Postal code 710049, P. R. China. He was with the School of Engineering, Institute for Digital Communications, The University of Edinburgh, Edinburgh, U.K.. The work of J. Xue is supported by the "Young Talent Support Plan" of Xi' an Jiaotong University and the UK EPSRC under grant number EP/L025299/1.

S. Biswas, and T. Ratnarajah are with the School of Engineering, Institute for Digital Communications, The University of Edinburgh, Edinburgh, EH9 3JL, U.K.. The work of S. Biswas and T. Ratnarajah are supported by the UK EPSRC under grant number EP/L025299/1, Massive MIMO for Future Wireless Communication Networks.

A. C. Cirik is with Ofinno Technologies, Herndon, VA 20171, United States.

$\mathrm{H}$. Du is with the College of Information Science and Technology, Jinan University, Guangzhou, China, and also with National Mobile Communications Research Laboratory, Southeast University. The work of Huiqin $\mathrm{Du}$ is supported by the National Natural Science Foundation of China (No.61401178) and the Open Research Fund of National Mobile Communications Research Laboratory Southeast University (No.2017D12).

Y. Yang is with the Interdisciplinary Centre for Security, reliability and trust, University of Luxembourg, L-1855 Luxembourg. The work of Yang is supported by the ERC project AGNOSTIC.

M. Sellathurai is with the Institute of Sensors, Signals \& Systems, School of Engineering \& Physical Sciences, Heriot-Watt University, U.K.. M. Sellathurai is supported by the UK EPSRC grant EP/M014126/1, Large Scale Antenna Systems Made Practical: Advanced Signal Processing for Compact Deployments and UGC -UKIERI 2016-17-058.

\section{INTRODUCTION}

Wireless data traffic has exponentially increased over the last decade and it is projected to increase even further. As a result, effective and efficient utilization of the scarce spectrum resources has become an extremely important issue. The currently deployed half-duplex (HD) wireless communication systems do not utilize the spectrum efficiently as transmission and reception happen orthogonally, either in time, denoted as time division duplexing (TDD), or in frequency, denoted as frequency division duplexing (FDD). Among the emerging technologies for next-generation wireless networks, fullduplex (FD) communication is considered as a way to potentially double the capacity of wireless communications. Simultaneous transmission and reception of overlapping signals in the same frequency had generally been assumed impossible in wireless communications due to the challenges involved in handling the self-interference [1], which is caused due to the signal received at the receive antennas of a FD node from its own transmitter antennas. However, thanks to the recent progress of cancellation made on self-interference suppression, FD communication systems have triggered enormous research interests [2], [3]. Consequently, FD is being considered as a key enabling technique for 5G and beyond systems [4], since it enables available spectral resources to be fully utilized in both time and frequency.

A single-antenna FD system was investigated in [5], while in [6], a multiple-antenna FD system was studied. Many feasible solutions including antenna, analog and digital cancellation have been demonstrated experimentally to mitigate the overwhelming self-interference, which is the fundamental challenge in implementing a full-duplex radio [7]-[10]. However, due to imperfect self-interference channel knowledge and hardware impairments in the transmitter chain, the selfinterference cannot be completely eradicated in practice. In this case, the performance is limited by the residual selfinterference, which is induced by the imperfection of the transmit and receive front-end chain [11]-[19]. In addition to self-interference, co-channel interference (CCI) from uplink (UL) to downlink (DL) nodes is another challenge in FD networks that needs to be overcome to fully exploit the multiaccess nature of the wireless medium in conjunction with FD systems. To optimize the system performance, self-interference and CCI in FD systems should be addressed jointly through beamforming [15], [20], [21].

It is a common practice in wireless communication devices 
that energy is supplied to an energy constrained wireless device with the help of rechargeable or replaceable batteries. However, these batteries have limited operation time and they need to be replaced or recharged frequently. Not only is this inconvenient, but also usually incurs high costs. According to the analyses in literature and the fact that radio signals can potentially carry wireless information and energy simultaneously, a new promising solution, energy harvesting $(\mathrm{EH})$ through simultaneous wireless information and power transfer (SWIPT), has been proposed [22]-[24]. This is seen as a key enabling technique for next generation wireless devices. In particular, the recent advent of low powered internet of things (IoT) devices and device to device (D2D) communications has made EH an interesting proposition. Such IoT/D2D devices will mainly make use of the current state of the art technologies such as bluetooth 5.0 low energy (LE), IEEE 802.11n, IEEE 802.11ac, etc., along with other future communication technologies such as mmWave transmission. The emergence of these low power consumption technologies makes it possible to realize wireless power transfer, which can act as a cornerstone for future green communications. This EH technique can be realized by two practical means, namely time-switching (TS) and power splitting (PS) which were introduced in [25]. The single-input single-output (SISO) system with additive white Gaussian noise (AWGN) was considered in [26], where a capacity-energy function to characterize the fundamental trade-off between wireless information and energy transmission was proposed. Moreover, SWIPT for multiple-antenna systems was studied in [27].

Further from a market perspective (Global Market Forecast (GMF) upto 2023), the EH system market by technology (light, vibration, RF, thermal), component, application (building and home automation, consumer electronics, IoT, industrial, transportation, security) is expected to witness a growth from USD $311.2 \mathrm{M}$ in 2016 to USD $645.8 \mathrm{M}$ by 2023 , at a CAGR of $10.62 \%$ between 2017 and 2023. The key players in this market are EnOcean GmbH (Germany), Mide Technology Corporation (US), Lord Microstrain (US); secondary battery and capacitor providers such as Cymbet Corporation (US), Linear Technologies (US), Murata Manufacturing Co. Ltd., (Japan), and Infinite Power Solution Inc. (US); power management IC manufacturers such as Linear Technologies (US), Cypress Semiconductor Corp. (US), STMicroelectronics (Switzerland), Texas Instruments (US), and Fujitsu (Japan).

Motivated by the potential of FD and $\mathrm{EH}$ in future wireless communication systems, such as IoT/D2D, in this paper we investigate the $\mathrm{EH}$ potential of multiple-input multipleoutput (MIMO) interference channels consisting of $K$ pairs of IoT/D2D FD nodes with SWIPT. The consideration of interference channel is suitable for IoT/D2D devices as multiple low powered devices will interact among each other in an IoT/D2D communication scenario. Each IoT/D2D node is assumed to have $N_{i}^{a}$ transmit and $M_{i}^{a}$ receive antennas, where $i \in\{1,2, \ldots, K\}$ and $a \in\{1,2\}$ and operates in FD mode. The consideration of FD and EH techniques at each node allows all the participating nodes to transmit/receive signal and harvest energy simultaneously. The harvested energy can then be used by the nodes to recharge their batteries without the need for external power supply ${ }^{1}$.

Indeed, the studied system in our paper shares many similarities to the traditional interference channel networks, both in terms of the system concept, service requirements, as well as the design guidelines. Nevertheless, the application of FD technology for $\mathrm{EH}$ in a IoT/D2D network introduces new fundamental challenges to the traditional interference channel models, which is the main focus of this paper. The main distinctions are summarized as follows:

- The EH requirements of an IoT network need to be revised when operating in a FD mode. This is because in a FD interference channel network, all the nodes share the same channel resource for transmission and reception, which results in the imposition of a higher interference intensity on the network. This issue becomes more critical considering the fact that the acquisition of an accurate CSI regarding the interference paths from the multiple users is relatively unrealistic, and calls for the consideration of a joint robust transmission strategy.

- In an FD MIMO interference channel network, the selfinterference at each node is a critical challenge, and strongly relates the performance/design of the UL/DL reception to the DL/UL transmission. In this respect, the consideration of an accurate transceiver model, including the impacts of transmission and reception distortions are critical, as it is well-established in the context of FD system design and analysis.

- In an FD MIMO interference channel network, apart from the self-interference, the interference paths among all nodes should be additionally taken into account. This impacts both the system performance, as well as the design strategy.

Note that the aforementioned considerations regarding the design of a robust FD multi-node MIMO IoT network, result in a relatively complicated problem structure. For perfect channel state information (CSI) at the nodes, the optimization problem is defined according to the SINR and EH constraints, which results in a non-convex optimization problem. When the CSI availability at the transmitters is imperfect, the errors of the CSI are assumed to be norm bounded, resulting in a semiinfinite problem.

Moreover, due to the transmit and receive distortions at the FD nodes in addition to imperfect CSI, EH and SINR become complicated functions, which make the transformation of the constraints in the optimization problems complicated. This, in turn, calls for a rigorous optimization and analysis, together with a dedicated computational complexity study. Numerical results demonstrate the feasibility of $\mathrm{EH}$ for FD. It is shown that the harvested energy can support small IoT devices with minimal power requirements to recharge their batteries.

Notations: The following notations are used in this paper. Matrices and vectors are denoted as bold capital and lowercase letters, respectively. $(\cdot)^{T}$ is the transpose, $(\cdot)^{H}$ represents the conjugate transpose, and $(\cdot)^{*}$ the conjugate. $\mathbb{E}\{\cdot\}$ denotes

\footnotetext{
${ }^{1}$ This paper does not focus on the modeling of charging of the battery. Analysis of the battery dynamics is beyond the scope of this paper. Interested readers can refer to [28], [29] for details on battery modelling.
} 


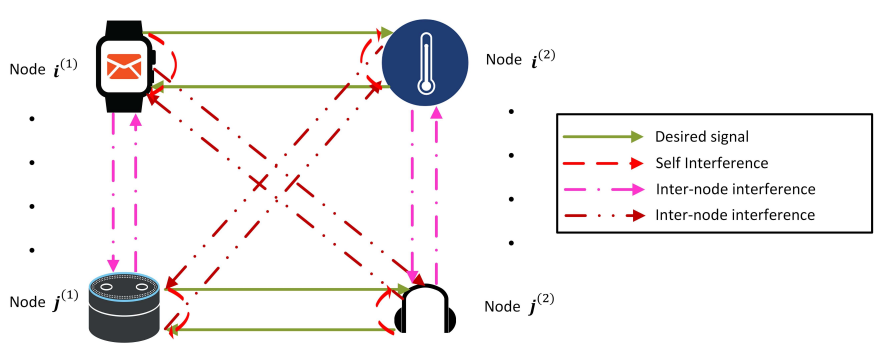

Fig. 1. An illustration of a bi-directional full-duplex MIMO interference channel involving $K$ pairs of IoT nodes.

the statistical expectation and $\operatorname{diag}(\mathbf{A})$ is the diagonal matrix with the same diagonal elements as $\mathbf{A}$. $\mathbf{I}_{N}$ is the $N$ by $N$ identity matrix, and $\operatorname{tr}\{\cdot\}$ is the trace operator. $\mathcal{C N}\left(\mu, \sigma^{2}\right)$ denotes a complex Gaussian distribution with mean $\mu$ and variance $\sigma^{2} . \mathbb{C}^{N \times M}$ denotes the set of complex matrices with a dimension of $N$ by $M .|\cdot|$ and $\|\cdot\|$ denote the absolute value and the Euclidean norm, respectively. The operator for multidimensional array is denoted by $\operatorname{vec}(\cdot) . \mathbf{A} \succeq \mathbf{0}$ indicates that matrix $\mathbf{A}$ is positive semidefinite, and $\operatorname{rank}(\mathbf{A})$ is the rank of matrix $\mathbf{A}$.

The rest of the paper is organized as follows. The system model is introduced in Section II. Section III and IV present the beamforming design problem for the MISO and MIMO interference channels with perfect CSI, respectively. The imperfect CSI case is presented in Section V followed by the numerical results in Section VI. Finally, Section VII provides the conclusion of the paper.

\section{SySTEM ModeL}

As shown in Fig. 1 on the top of next page, the system under consideration consists of $K$ pairs of FD IoT nodes, where each IoT node pair exchanges information simultaneously in a two way communication. For simplification, we assume that the MIMO FD IoT nodes in the $i$ th link have $N_{i}$ transmit antennas and $M_{i}$ receive antennas.

The node $i^{(a)}$, where $i \in\{1, \ldots, K\}$ and $a \in\{1,2\}$ receives signals from all the transmitters in the system. $\mathbf{H}_{i i}^{(a b)} \in$ $\mathbb{C}^{M_{i} \times N_{i}}$ is the desired channel between node $a$ and $b$ of the $i$ th transmitter-receiver pair, where $b \in\{1,2\}$ and $b \neq a$. The self-interference channel of the node $i^{(a)}$ is denoted as $\mathbf{H}_{i i}^{(a a)} \in \mathbb{C}^{M_{i} \times N_{i}}, a \in\{1,2\}$ and the inter-user interference channel from the transmitter antennas of the node $c$ in the $j$ th pair to the receiver antenna of the node $a$ in the $i$ th pair, $(i, j) \in\{1, \ldots, K\}$ and $j \neq i$ is denoted as $\mathbf{H}_{i j}^{(a c)} \in$ $\mathbb{C}^{M_{i} \times N_{j}},(a, c) \in\{1,2\}$.

Hereinafter, different mathematical methods will be applied to obtain optimal EH solutions for both MISO and MIMO FD systems. We begin our analysis by considering the perfect CSI MISO and MIMO cases, which are then extended to MISO and MIMO cases with imperfect CSI later in the paper.

\section{Full Duplex Systems With PERfeCt CSI}

In this section, we assume that perfect CSI is available at all the IoT nodes. The MISO scenario will be investigated first followed by the MIMO case ${ }^{2}$.

\section{A. Transceiver design for MISO interference channel}

The transmitted data stream of node $i^{(a)}$ is denoted as $d_{i}^{(a)}, i \in\{1, \ldots, K\}, a \in\{1,2\}$, and is assumed to be complex, zero mean, independent and identically distributed (i.i.d.) with unit variance. The $N_{i} \times 1$ signal vector transmitted by node $i^{(a)}$ is given by

$$
\mathbf{x}_{i}^{(a)}=\mathbf{v}_{i}^{(a)} d_{i}^{(a)}, \quad i=1, \ldots, K, a \in\{1,2\},
$$

where $\mathbf{v}_{i}^{(a)} \in \mathbb{C}^{N_{i} \times 1}$ represents the precoding vector.

The received signal at node $i^{(a)}$ is a combination of the signals transmitted by all the IoT nodes plus the additive noise, which is written as

$$
\begin{aligned}
y_{i}^{(a)}= & \left(\mathbf{h}_{i i}^{(a b)}\right)^{H}\left(\mathbf{x}_{i}^{(b)}+\mathbf{c}_{i}^{(b)}\right)+\left(\mathbf{h}_{i i}^{(a a)}\right)^{H}\left(\mathbf{x}_{i}^{(a)}+\mathbf{c}_{i}^{(a)}\right) \\
& +\sum_{j \neq i}^{K} \sum_{c=1}^{2}\left(\mathbf{h}_{i j}^{(a c)}\right)^{H}\left(\mathbf{x}_{j}^{(c)}+\mathbf{c}_{j}^{(c)}\right)+e_{i}^{(a)} \\
& +n_{i}^{(a)}, i \in\{1, \ldots, K\}, \quad(a, b) \in\{1,2\}, a \neq b .(2)
\end{aligned}
$$

Here, $n_{i}^{(a)}$ is the additive white Gaussian noise (AWGN) at node $i^{(a)}$ with zero mean and variance $\sigma_{n}^{2}$.

In (2), $\mathbf{c}_{i}^{(a)} \in \mathbb{C}^{N_{i}}, i \in\{1, \ldots, K\}, a \in\{1,2\}$ is the noise at the transmitter antennas of node $i^{(a)}$, which models the effect of limited transmitter dynamic range (DR) and closely approximates the effects of additive power-amplifier noise, non-linearities in the DAC and phase noise [12]. The mean of $\mathbf{c}_{i}^{(a)}$ is $\mathbf{0}$, and the variance of $\mathbf{c}_{i}^{(a)}$ is proportional to the energy of the intended signal at each transmit antenna, i.e.,

$$
\mathbf{c}_{i}^{(a)} \sim \mathcal{C N}\left(\mathbf{0}, \kappa \operatorname{diag}\left(\mathbf{v}_{i}^{(a)}\left(\mathbf{v}_{i}^{(a)}\right)^{H}\right)\right), \mathbf{c}_{i}^{(a)} \perp \mathbf{x}_{i}^{(a)},
$$

where $\perp$ denotes the statistical independence ${ }^{3}$.

In (2), $e_{i}^{(a)}, i \in\{1, \ldots, K\}, a \in\{1,2\}$ is the additive distortion at the receiver antenna of node $i^{(a)}$, which models the effect of limited receiver DR and closely approximates the combined effects of additive gain-control noise, non-linearities in the ADC and phase noise. The mean of $e_{i}^{(a)}$ is 0 and the variance is proportional to the energy of the undistorted received signal at the receive antenna. In particular, $e_{i}^{(a)}$ is modeled as

$$
e_{i}^{(a)} \sim \mathcal{C N}\left(0, \beta \Phi_{i}^{(a)}\right), e_{i}^{(a)} \perp u_{i}^{(a)},
$$

where $\Phi_{i}^{(a)}=\operatorname{Var}\left\{u_{i}^{(a)}\right\}$ is the variance of $u_{i}^{(a)}$, and $u_{i}^{(a)}$ is the undistorted received signal at the node $i^{(a)}$, i.e., $u_{i}^{(a)}=$ $y_{i}^{(a)}-e_{i}^{(a)}$.

\footnotetext{
${ }^{2}$ The consideration of both the MISO and MIMO cases is due to the fact that the MIMO case requires certain approximations to derive the covariance matrix of the signal. However, the MISO case doesn't require any such approximations and hence the MISO case may not be a straigtforward extension of the MIMO case in our analysis.

${ }^{3}$ Considering the measurements of various hardware setups which were shown in [30], [31], the received signal modeled as (2) closely approximates the combined effects of additive power-amp noise, non-linearities in the DAC and power-amp, and oscillator phase noise. Meanwhile, by the definition of limited dynamic range, the transmitter-noise variance is dependent on intended signal power [16].
} 


$$
\begin{aligned}
\Sigma_{i}^{(a)} & =\left(\mathbf{h}_{i i}^{(a b)}\right)^{H}\left(\kappa(1+\beta) \operatorname{diag}\left(\mathbf{v}_{i}^{(b)}\left(\mathbf{v}_{i}^{(b)}\right)^{H}\right)+\beta \mathbf{v}_{i}^{(b)}\left(\mathbf{v}_{i}^{(b)}\right)^{H}\right)\left(\mathbf{h}_{i i}^{(a b)}\right) \\
& +\left(\mathbf{h}_{i i}^{(a a)}\right)^{H}\left(\kappa(1+\beta) \operatorname{diag}\left(\mathbf{v}_{i}^{(a)}\left(\mathbf{v}_{i}^{(a)}\right)^{H}\right)+\beta \mathbf{v}_{i}^{(a)}\left(\mathbf{v}_{i}^{(a)}\right)^{H}\right)\left(\mathbf{h}_{i i}^{(a a)}\right) \\
& +\sum_{j \neq i}^{K} \sum_{c=1}^{2}(1+\beta)\left[\left(\mathbf{h}_{i j}^{(a c)}\right)^{H}\left(\mathbf{v}_{j}^{(c)}\left(\mathbf{v}_{j}^{(c)}\right)^{H}+\kappa \operatorname{diag}\left(\mathbf{v}_{j}^{(c)}\left(\mathbf{v}_{j}^{(c)}\right)^{H}\right)\right)\left(\mathbf{h}_{i j}^{(a c)}\right)\right]+(1+\beta) \sigma_{n}^{2}
\end{aligned}
$$

Node $i^{(a)}$ has the knowledge of the interfering codewords $\mathbf{x}_{i}^{(a)}$ and the channel $\mathbf{h}_{i i}^{(a)}$. So the self-interference term $\left(\mathbf{h}_{i i}^{(a a)}\right)^{T} \mathbf{x}_{i}^{(a)}$ is known, and thus can be canceled [12]. The received signal after self-interference cancellation can then be written as

$$
\begin{aligned}
\tilde{y}_{i}^{(a)} & =y_{i}^{(a)}-\left(\mathbf{h}_{i i}^{(a a)}\right)^{T} \mathbf{x}_{i}^{(a)} \\
& =\left(\mathbf{h}_{i i}^{(a b)}\right)^{T} \mathbf{x}_{i}^{(b)}+v_{i}^{(a)}
\end{aligned}
$$

where $v_{i}^{(a)}$ is the unknown interference-plus noise component after self-interference cancellation, and is given by

$$
\begin{aligned}
v_{i}^{(a)}= & \left(\mathbf{h}_{i i}^{(a b)}\right)^{T} \mathbf{c}_{i}^{(b)}+\left(\mathbf{h}_{i i}^{(a a)}\right)^{T} \mathbf{c}_{i}^{(a)}+e_{i}^{(a)}+n_{i}^{(a)} \\
& +\sum_{j \neq i}^{K} \sum_{c=1}^{2}\left(\mathbf{h}_{i j}^{(a c)}\right)^{T}\left(\mathbf{x}_{j}^{(c)}+\mathbf{c}_{j}^{(c)}\right) .
\end{aligned}
$$

Now, using (3)-(4), $\Sigma_{i}^{(a)}$, the variance of $v_{i}^{(a)}$ is given as (7).

By means of a power splitter, the received signal is now divided into two parts, one for the information decoder and another for EH. Let $\rho_{i}^{(a)}$ denote the PS ratio for receiver $i^{(a)}$, which means that a portion $\rho_{i}^{(a)}$ of the signal power is used for signal detection while the remaining portion $1-\rho_{i}^{(a)}$ is diverted to an energy harvester. Accordingly, the available signal for information decoding at receiver $i^{(a)}$ can be expressed as

$$
r_{i}^{(a)}=\sqrt{\rho_{i}^{(a)}} \tilde{y}_{i}^{(a)}+m_{i}^{(a)},
$$

where $m_{i}^{(a)}$ is the additional AWGN circuit noise with zero mean and variance $\sigma_{i_{(a)}}^{2}$ due to phase offset and non-linearities during baseband conversion [32]. Using (8), the SINR at receiver $i^{(a)}$ is given by

$$
\operatorname{SINR}_{i}^{(a)}=\frac{\rho_{i}^{(a)}\left|\left(\mathbf{h}_{i i}^{(a b)}\right)^{T} \mathbf{v}_{i}^{(b)}\right|^{2}}{\rho_{i}^{(a)} \Sigma_{i}^{(a)}+\sigma_{i_{(a)}}^{2}} .
$$

Besides, the total harvested energy that can be stored by receiver $i^{(a)}$ is given as

$$
\begin{aligned}
\mathrm{EH}_{i}^{(a)}= & \xi_{i}^{(a)}\left(1-\rho_{i}^{(a)}\right) \mathbb{E}\left\{\left|y_{i}^{(a)}\right|^{2}\right\} \\
= & \xi_{i}^{(a)}\left(1-\rho_{i}^{(a)}\right)\left(\left|\left(\mathbf{h}_{i i}^{(a b)}\right)^{T} \mathbf{v}_{i}^{(b)}\right|^{2}\right. \\
& \left.+\left|\left(\mathbf{h}_{i i}^{(a)}\right)^{T} \mathbf{v}_{i}^{(a)}\right|^{2}+\Sigma_{i}^{(a)}\right)
\end{aligned}
$$

where $\xi_{i}^{(a)} \in(0,1)$ denotes the energy conversion efficiency of the $i^{(a)}$ th $\mathrm{EH}$ unit.
We focus on transmit filter and PS ratio design, in order to minimize the total transmitted power, subject to SINR and EH constraints. The optimization scheme is formulated as follows.

$$
\begin{array}{cl}
\min _{\substack{(b) \\
\mathbf{v}_{i}^{(b)}, \rho_{i}^{(a)}}} & \sum_{i=1}^{K} \sum_{b=1}^{2}\left\|\mathbf{v}_{i}^{(b)}\right\|^{2} \\
\text { s.t. } & \operatorname{SINR}_{i}^{(a)} \geq \gamma_{i}^{(a)}, \forall(i, a), \\
& \operatorname{EH}_{i}^{(a)} \geq \delta_{i}^{(a)}, 0 \leq \rho_{i}^{(a)} \leq 1, \forall(i, a),
\end{array}
$$

where $\gamma_{i}^{(a)}$ and $\delta_{i}^{(a)}$ are the SINR and EH thresholds at the $i^{(a)}$ th IoT receiver, respectively. The EH constraint represents the minimum amount of energy required in order to ensure that sufficient amount of energy is harvested in each transmission time. To obtain the optimal solution, the sufficient conditions to obtain a feasible solution and the procedure and techniques will be discussed in the following sections.

\section{B. Extension to MIMO Interference Channel}

In this section, we extend the model proposed in the previous section to multiple-antenna IoT receivers, where the nodes at the $i$ th link now has $M_{i}$ receive antennas. Note that the channel vectors $\mathbf{h}_{i j}^{(a c)}, \forall(i, j, a, c)$ in the MISO case is now replaced with the channel matrices $\mathbf{H}_{i j}^{(a c)}, \forall(i, j, a, c)$ for the MIMO case. Accordingly, for this case, the $\operatorname{SINR}_{i}^{(a)}$ and $\mathrm{EH}_{i}^{(a)}$ are defined as

$$
\begin{aligned}
\operatorname{SINR}_{i}^{(a)}= & \frac{\rho_{i}^{(a)}\left|\left(\mathbf{u}_{i}^{(a)}\right)^{H} \mathbf{H}_{i i}^{(a b)} \mathbf{v}_{i}^{(b)}\right|^{2}}{\rho_{i}^{(a)}\left(\mathbf{u}_{i}^{(a)}\right)^{H} \mathbf{\Sigma}_{i}^{(a)} \mathbf{u}_{i}^{(a)}+\sigma_{i_{(a)}}^{2}\left\|\mathbf{u}_{i}^{(a)}\right\|^{2}}, \\
\mathrm{EH}_{i}^{(a)}= & \xi_{i}^{(a)}\left(1-\rho_{i}^{(a)}\right) \operatorname{tr}\left\{\mathbf{H}_{i i}^{(a b)} \mathbf{v}_{i}^{(b)}\left(\mathbf{v}_{i}^{(b)}\right)^{H}\left(\mathbf{H}_{i i}^{(a b)}\right)^{H}\right. \\
& \left.+\mathbf{H}_{i i}^{(a a)} \mathbf{v}_{i}^{(a)}\left(\mathbf{v}_{i}^{(a)}\right)^{H}\left(\mathbf{H}_{i i}^{(a a)}\right)^{H}+\mathbf{\Sigma}_{i}^{(a)}\right\} .(13)
\end{aligned}
$$

In (12) and (13), $\boldsymbol{\Sigma}_{i}^{(a)}$ is the covariance matrix of the total interference-plus noise components, which is approximated by ignoring the terms that include $\kappa \beta \ll 1$, and is expressed as $(14)^{4}$

\footnotetext{
${ }^{4}$ Note that approximation of $\boldsymbol{\Sigma}_{i}^{(a)}$ is a practical assumption [12], as the terms $\kappa$ and $\beta$ are much smaller than 1 .
} 


$$
\begin{aligned}
\boldsymbol{\Sigma}_{i}^{(a)} \approx & \kappa \mathbf{H}_{i i}^{(a b)} \operatorname{diag}\left(\mathbf{v}_{i}^{(b)}\left(\mathbf{v}_{i}^{(b)}\right)^{H}\right)\left(\mathbf{H}_{i i}^{(a b)}\right)^{H}+\kappa \mathbf{H}_{i i}^{(a a)} \operatorname{diag}\left(\mathbf{v}_{i}^{(a)}\left(\mathbf{v}_{i}^{(a)}\right)^{H}\right)\left(\mathbf{H}_{i i}^{(a a)}\right)^{H} \\
& +\sum_{j \neq i}^{K} \sum_{c=1}^{2}\left[\mathbf{H}_{i j}^{(a c)}\left(\mathbf{v}_{j}^{(c)}\left(\mathbf{v}_{j}^{(c)}\right)^{H}+\kappa \operatorname{diag}\left(\mathbf{v}_{j}^{(c)}\left(\mathbf{v}_{j}^{(c)}\right)^{H}\right)\right)\left(\mathbf{H}_{i j}^{(a c)}\right)^{H}\right] \\
& +\sum_{j=1}^{K} \sum_{c=1}^{2} \beta \operatorname{diag}\left(\mathbf{H}_{i j}^{(a c)} \mathbf{v}_{j}^{(c)}\left(\mathbf{v}_{j}^{(c)}\right)^{H}\left(\mathbf{H}_{i j}^{(a c)}\right)^{H}\right)+\sigma_{n}^{2} \mathbf{I}_{M_{i}} .
\end{aligned}
$$

Now, the optimization problem (11) can be reformulated for the MIMO case, as

$$
\begin{array}{cl}
\min _{\mathbf{v}_{i}^{(b)}, \mathbf{u}_{i}^{(a)}, \rho_{i}^{(a)}} & \sum_{i=1}^{K} \sum_{b=1}^{2}\left\|\mathbf{v}_{i}^{(b)}\right\|^{2} \\
\text { s.t. } & \operatorname{SINR}_{i}^{(a)} \geq \gamma_{i}^{(a)}, \forall(i, a), \\
& \operatorname{EH}_{i}^{(a)} \geq \delta_{i}^{(a)}, \forall(i, a), \\
& 0 \leq \rho_{i}^{(a)} \leq 1, \\
& \left\|\mathbf{u}_{i}^{(a)}\right\|^{2}=1, \forall(i, a),
\end{array}
$$

where $\mathbf{u}_{i}^{(a)} \in \mathbb{C}^{M_{i} \times 1}, i \in\{1, \ldots, K\}, a \in\{1,2\}$ is the linear receiver applied at the receiver $i^{(a)}$.

Problem (15) is non-convex and difficult to solve due to the quadratic terms involving all transmit beamforming vectors. In order to solve this problem, we introduce a new variable $\mathbf{W}_{i}^{(b)} \triangleq \mathbf{v}_{i}^{(b)}\left(\mathbf{v}_{i}^{(b)}\right)^{H}, \forall(i, b)$ with $\operatorname{rank}\left(\mathbf{W}_{i}^{(b)}\right)=1$, and relax the corresponding problem by dropping the rank constraint. The reformulated problem can be expressed as

$$
\begin{array}{cl}
\underset{\mathbf{W}_{i}^{(b)}, \mathbf{u}_{i}^{(a)}, \rho_{i}^{(a)}}{\min } & \sum_{i=1}^{K} \sum_{b=1}^{2} \operatorname{tr}\left\{\mathbf{W}_{i}^{(b)}\right\} \\
\text { s.t. } & A-\frac{\sigma_{i_{(a)}^{2}}^{2}\left\|\mathbf{u}_{i}^{(a)}\right\|^{2}}{\rho_{i}^{(a)}} \geq 0, \\
& B-\frac{\left(\delta_{i}^{(a)}\right)^{2}}{1-\rho_{i}^{(a)}} \geq 0, \\
& 0 \leq \rho_{i}^{(a)} \leq 1, \\
& \mathbf{W}_{i}^{(a)} \succeq 0, \forall(i, a),
\end{array}
$$

where the variables $A$ and $B$ are obtained after performing some simple algebraic manipulations, and are defined as

$$
\begin{aligned}
A= & \frac{1}{\gamma_{i}^{(a)}}\left(\left(\mathbf{u}_{i}^{(a)}\right)^{H} \mathbf{H}_{i i}^{(a b)} \mathbf{W}_{i}^{(b)}\left(\mathbf{H}_{i i}^{(a b)}\right)^{H} \mathbf{u}_{i}^{(a)}\right) \\
& -\left(\mathbf{u}_{i}^{(a)}\right)^{H} \boldsymbol{\Sigma}_{i}^{(a)} \mathbf{u}_{i}^{(a)}, \\
B= & \xi_{i}^{(a)} \operatorname{tr}\left\{\mathbf{H}_{i i}^{(a b)} \mathbf{v}_{i}^{(b)}\left(\mathbf{v}_{i}^{(b)}\right)^{H}\left(\mathbf{H}_{i i}^{(a b)}\right)^{H}\right. \\
& \left.+\mathbf{H}_{i i}^{(a a)} \mathbf{v}_{i}^{(a)}\left(\mathbf{v}_{i}^{(a)}\right)^{H}\left(\mathbf{H}_{i i}^{(a a)}\right)^{H}+\boldsymbol{\Sigma}_{i}^{(a)}\right\} .
\end{aligned}
$$

To obtain the optimal $\mathbf{v}_{i}^{(b)}$, we propose an iterative alternating algorithm given on top of this page to solve problem (16). Some insightful discussion for the original problem and its solution is given in the following.

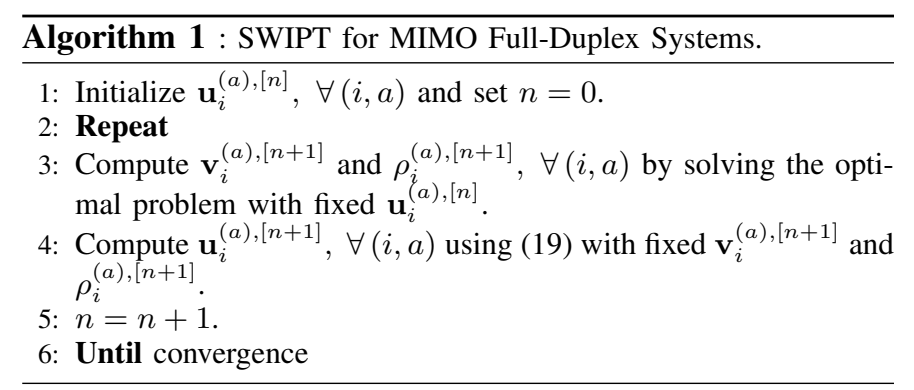

Finding the optimal $\mathbf{V}$ : First, under fixed $\mathbf{u}_{i}^{(a)}$, we drop the constraint $\operatorname{rank}\left(\mathbf{W}_{i}^{(b)}\right)=1$ for numerical tractability to solve for the optimal $\mathbf{W}_{i}^{(b)}$ and $\rho_{i}^{(a)}$. Note that, the rank of the solution $\mathbf{W}_{i}^{(b)}$ can be guaranteed as rank one in general, and the randomization procedure is proposed to generate a feasible but suboptimal solution. Suppose $\mathbf{W}^{*}$ is the optimal solution to the relaxed problem. Due to the relaxation, $\operatorname{rank}\left(\mathbf{W}^{*}\right)$ may not satisfy (15) in general. As a result, we adopt the randomization technique [1], [2], in which the solution $\mathbf{W}^{*}$ is eigen-decomposed as $\mathbf{W}^{*}=\mathbf{U}_{\mathbf{W}^{*}} \boldsymbol{\Lambda}_{\mathbf{W}^{*}} \mathbf{U}_{\mathbf{W}^{*}}^{H}$ and the solution to (15) is chosen as $\mathbf{W}=\mathbf{U}_{\mathbf{W}^{*}} \boldsymbol{\Lambda}_{\mathbf{W}^{*}}^{1 / 2} \mathbf{V v}^{H} \Lambda_{\mathbf{W}^{*}}^{1 / 2} \mathbf{U}_{\mathbf{W}^{*}}^{H}$ with $N_{i} \times 1$ uniform or Gaussian distributed random vector $\mathbf{v}$ [33], [34].

Secondly, under fixed $\mathbf{v}_{i}^{(b)}$ and $\rho_{i}^{(a)}$, the problem to compute the optimal $\mathbf{u}_{i}^{(a)}$ boils down to a feasibility problem, which has a closed form solution, and it is given by [16], [35]

$$
\mathbf{u}_{i}^{(a)}=\frac{\left(\rho_{i}^{(a)} \boldsymbol{\Sigma}_{i}^{(a)}+\sigma_{i_{(a)}}^{2} \mathbf{I}_{M_{i}}\right)^{-1} \mathbf{H}_{i i}^{(a b)} \mathbf{v}_{i}^{(b)}}{\left\|\left(\rho_{i}^{(a)} \boldsymbol{\Sigma}_{i}^{(a)}+\sigma_{i_{(a)}}^{2} \mathbf{I}_{M_{i}}\right)^{-1} \mathbf{H}_{i i}^{(a b)} \mathbf{v}_{i}^{(b)}\right\|} .
$$
1.

The steps of the proposed algorithm are shown in Algorithm

Remark: Since the sum transmission power decreases (or stays at the same value) at each iteration, and it is lower bounded by zero, the sum transmission power will converge. But since the primal problem (15) is non-convex, global optimality is not guaranteed.

\section{Complexity Analysis}

In this subsection, we discuss the computational complexity of the proposed algorithm. The number of arithmetic operations required to solve a standard real-valued SDP problem

$$
\begin{array}{ll}
\min _{\mathbf{x} \in \mathcal{R}^{n}} & \mathbf{c}^{T} \mathbf{x} \\
\text { subject to } & \mathbf{A}_{0}+\sum_{i=1}^{n} x_{i} \mathbf{A}_{i} \succeq \mathbf{0}, \text { and } \quad\|\mathbf{x}\|_{2} \leq X,
\end{array}
$$


where $\mathbf{A}_{i}$ denotes the symmetric block-diagonal matrices with $P$ diagonal blocks of size $e_{l} \times e_{l}, l=1, \ldots, P$, is upperbounded by [36]

$$
\mathcal{O}(1)\left(1+\sum_{l=1}^{P} e_{l}\right)^{1 / 2} n\left(n^{2}+n \sum_{l=1}^{P} e_{l}^{2}+\sum_{l=1}^{P} e_{l}^{3}\right) .
$$

The main computational complexity of Algorithm 1 arises from computing the optimal $\mathbf{v}_{i}^{(a)}$ and $\mathbf{u}_{i}^{(a)}$. For simplicity, let us assume same number of transmit and receive antennas at each node, i.e., $M_{i}=M$ and $N_{i}=N, i=1, \ldots, K$. Accordingly the complexity analysis is given as below.

1) Computations required to calculate $\mathbf{v}_{i}^{(a)}$ : Since the proposed algorithm solves a SDP problem in Step 2, the number of arithmetic operations required to compute optimal $\mathbf{v}_{i}$ is calculated from (22) as follows. In computing $\mathbf{v}_{i}$, the number of diagonal blocks $P$ is equal to $8 K$. For the constraint (16b) for each node, the dimension of blocks are $e_{i}^{(a)}=M+1, i=1, \ldots, K, a \in\{1,2\}$. For the constraint (16c), $e_{i}^{(a)}=3 M^{2}+1, i=1, \ldots, K, a \in\{1,2\}$. For the constraint (16d), and (16e), $e_{i}^{(a)}=1$, and $e_{i}^{(a)}=N^{2}, i=$ $1, \ldots, K, a \in\{1,2\}$, respectively. The unknown variables to be determined are of size $n=2 N^{2}+2 M+1$, where the first and second terms correspond to the real and imaginary parts of $\mathbf{W}_{i}^{(a)}$ and $\mathbf{u}_{i}^{(a)}$, respectively, while the third term represents the slack variable.

2) Computations required to calculate $\mathbf{u}_{i}^{(a)}$ : The computation of the receive beamformer is calculated from (19) as follows [37].

- Term in numerator inside the inverse: $2 \sum_{j=1}^{K} N\left(N+\left(1-\frac{N+1}{2}\right)\right)+2 M^{2}$

- Term in numerator outside the inverse: $2 M N-M$

- Inverse term in numerator: $M^{3}+M^{2}+M$

- Product of the terms in numerator (outside the inverse and the inverse): $2 M^{2}-M$

Accordingly, the total computational complexity to calculate the receiver matrix is in the order of $\mathcal{O}\left(\phi\left(K^{2} X+K M^{2}(M+\right.\right.$ $5))$ ), where $X=2 N\left(N+\left(1-\frac{N+1}{2}\right)\right)$.

\section{Transceiver Design With IMPERFECT CSI}

Considering a more realistic scenario, it may not be possible to obtain perfect CSI at all the IoT nodes due to, for example error of channel estimation, quantization errors, feedback delay, etc. Hence, it is necessary and important to optimize the system under imperfect CSI and design robust transceivers. In this section, we will investigate the FD MISO and MIMO interference channels again, but with the important discrepancy that the channels are now imperfectly known at the IoT nodes.

\section{A. FD MISO system with imperfect CSI}

Considering the well-known norm-bounded error (NBE) model [38], the actual CSI from the transmitter antennas of node $b$ in the $j$ th tier to the receiver antenna of node $a$ in the $i$ th tier is given by

$$
\begin{aligned}
\mathbf{h}_{i j}^{(a b)} & =\widetilde{\mathbf{h}}_{i j}^{(a b)}+\mathbf{e}_{i j}^{a b}, \\
(i, j) & \in\{1, \ldots, K\} \text { and }(a, b) \in\{1,2\},
\end{aligned}
$$

where $\widetilde{\mathbf{h}}_{i j}^{(a b)}$ is the estimated channel vector and $\mathbf{e}_{i j}^{a b}$ denotes the CSI error vector, which is bounded by its known positive constant Euclidean norm as

$$
\left\|\mathbf{e}_{i j}^{a b}\right\| \leq \eta_{i j}^{a b},(i, j) \in\{1, \ldots, K\} \text { and }(a, b) \in\{1,2\} .
$$

In this case, the uncertainty set of $\mathbf{h}_{i j}^{(a b)}$ can be defined as

$$
\begin{aligned}
& \mathcal{S}_{i j}^{(a b)}=\left\{\mathbf{h} \mid \mathbf{h}=\widetilde{\mathbf{h}}_{i j}^{(a b)}+\mathbf{e}_{i j}^{a b}, \quad\left\|\mathbf{e}_{i j}^{a b}\right\| \leq \eta_{i j}^{a b}\right\}, \\
& (i, j) \in\{1, \ldots, K\} \text { and }(a, b) \in\{1,2\} .
\end{aligned}
$$

Accordingly, we can rewrite the optimization problem in (11) as

$$
\begin{array}{cl}
\min _{\mathbf{v}_{i}^{(b)}, \rho_{i}^{(a)}} & \sum_{i=1}^{K} \sum_{b=1}^{2}\left\|\mathbf{v}_{i}^{(b)}\right\|^{2} \\
\text { s.t. } & \operatorname{SINR}_{i}^{(a)} \geq \gamma_{i}^{(a)}, \forall(i, a), \\
& \operatorname{EH}_{i}^{(a)} \geq \delta_{i}^{(a)}, 0 \leq \rho_{i}^{(a)} \leq 1, \forall(i, a), \\
& \mathbf{h}_{i j}^{(a b)}=\widetilde{\mathbf{h}}_{i j}^{(a b)}+\mathbf{e}_{i j}^{a b}, \\
& \left\|\mathbf{e}_{i j}^{a b}\right\|^{2} \leq \eta_{i j}^{a b^{2}}, \\
& (i, j) \in\{1, \ldots, K\} \text { and }(a, b) \in\{1,2\},
\end{array}
$$

where $\operatorname{SINR}_{i}^{(a)}$ and $\mathrm{EH}_{i}^{(a)}$ are given by (9) and (10), respectively. Now, introducing several auxiliary variables and applying the S-procedure [39] lemma, the problem for the MISO case can be expressed as (28), detailed steps of which are included in Appendix A. In (28), $\lambda_{i j}^{a b}$ and $\mu_{i j}^{a b}$ are slack variables for $(i, j) \in\{1, \ldots, K\}$ and $(a, b) \in\{1,2\}$, and variables $a_{i i}^{a b}, b_{i i}^{a a}, c_{i j}^{a c}, \widehat{a}_{i i}^{a b}, \widehat{b}_{i i}^{a a}, \widehat{c}_{i j}^{a c}$ and $d_{i i}^{(a a)}$ are defined in (32), (33), (34), (40), (41), (42) and (43) in Appendix A respectively. The above problem can be solved iteratively by using the standard CVX toolbox, a package in Matlab for specifying and solving convex programs [40], [41].

\section{B. FD MIMO system with imperfect CSI}

Similarly, for the MIMO scenario the actual CSI from the transmitter antennas of the node $b$ in the $j$ th tier to the receiver antennas of the node $a$ in the $i$ th tier can be expressed as

$$
\begin{aligned}
& \mathbf{H}_{i j}^{(a b)}=\widetilde{\mathbf{H}}_{i j}^{(a b)}+\mathbf{E}_{i j}^{a b}, \\
& (i, j) \in\{1, \ldots, K\} \text { and }(a, b) \in\{1,2\},
\end{aligned}
$$

where $\widetilde{\mathbf{H}}_{i j}^{(a b)}$ is the estimated channel matrix and $\mathbf{E}_{i j}^{a b}$ denotes the CSI error matrix, which is bounded by its known positive constant Frobenius norm as

$$
\left\|\mathbf{E}_{i j}^{a b}\right\|_{F} \leq \eta_{i j}^{a b},(i, j) \in\{1, \ldots, K\} \text { and }(a, b) \in\{1,2\} .
$$

Now reformulating the SINR and $\mathrm{EH}$ constraints and introducing slack variables, the problem for the MIMO case can be expressed as (31), the lengthy proof of which is relegated to Appendix $B$. The variables $a_{i i}^{a b}, b_{i i}^{a a}, c_{i j}^{a c}, d_{i i}^{(a a)}, \bar{a}_{i i}^{a a}, \widehat{a}_{i i}^{a b}, \widehat{b}_{i i}^{a a}, \widehat{c}_{i j}^{a c}$ and $\widehat{d}_{i i}^{(a a)}$ are defined in (69), (70), (71), (72), (80), (81), (82), (83) and (84) in Appendix B, respectively. The optimal solutions can now be obtained via an alternating minimization approach. 


$$
\begin{array}{cl}
\underset{\mathbf{W}_{i}^{(b)}, \rho_{i}^{(a)}, \lambda_{i j}^{a b}, \mu_{i j}^{a b}, a_{i i}^{a b},}{\min _{i i}, c_{i j}^{a c}, \widehat{a}_{i i}^{a b}, \widehat{b}_{i i}^{a a}, \widehat{c}_{i j}^{a c}, d_{i i}^{(a a)}} & \sum_{i=1}^{2} \operatorname{tr}\left\{\mathbf{W}_{i}^{(b)}\right\} \\
\text { s.t. } & (50),(51),(52),(53),(57), \\
& (58),(59),(60),(61), \\
& \mathbf{W}_{i}^{(b)} \succeq 0,0 \leq \rho_{i}^{(a)} \leq 1, \\
& \lambda_{i j}^{a b} \geq 0, \mu_{i j}^{a b} \geq 0, \\
& a_{i i}^{a b} \geq 0, b_{i i}^{a a} \geq 0, \\
& c_{i j}^{a c} \geq 0, \widehat{a}_{i i}^{a b} \geq 0, \\
& \widehat{b}_{i i}^{a a} \geq 0, \widehat{c}_{i j}^{a c} \geq 0, d_{i i}^{(a a)} \geq 0, \\
& (i, j) \in\{1, \ldots, K\} \text { and }(a, b, c) \in\{1,2\},
\end{array}
$$

$$
\begin{gathered}
\min \\
\mathbf{W}_{i}^{(b)}, \rho_{i}^{(a)}, \lambda_{i j}^{a b}, \mu_{i j}^{a b}, a_{i i}^{a b}, b_{i i}^{a a}, \\
c_{i j}^{a c}, d_{i i}^{(a a)} \bar{a}_{i i}^{a a}, \widehat{a}_{i i}^{a b}, \widehat{b}_{i i}^{a a}, \widehat{c}_{i j}^{a c}, \widehat{d}_{i i}^{(a a)}
\end{gathered}
$$$$
\sum_{i=1}^{K} \sum_{b=1}^{2} \operatorname{tr}\left\{\mathbf{W}_{i}^{(b)}\right\}
$$

s.t.

(63), (64), (65), (66), (67), (68),

(73), (74), (75), (76), (77), (78), (79),

$\mathbf{W}_{i}^{(b)} \succeq 0,0 \leq \rho_{i}^{(a)} \leq 1$,

$\lambda_{i j}^{a b} \geq 0, \mu_{i j}^{a b} \geq 0$,

$a_{i i}^{a b} \geq 0, b_{i i}^{a a} \geq 0$

$c_{i j}^{a c} \geq 0, d_{i i}^{(a a)} \geq 0$,

$\bar{a}_{i i}^{a a} \geq 0, \widehat{a}_{i i}^{a b} \geq 0$

$\widehat{b}_{i i}^{a a} \geq 0, \widehat{c}_{i j}^{a c} \geq 0, \widehat{d}_{i i}^{a a} \geq 0$,

$(i, j) \in\{1, \ldots, K\}$ and $(a, b, c) \in\{1,2\}$.

Remark: By solving the problem (31), the matrices $\mathbf{W}_{i}^{(b)}$ are not guaranteed to be of rank one mathematically. Generally speaking, the solution provides a lower bound to the original problem. As we mentioned before, it is worth noting that our solutions are of rank one in most cases, which means the principal eigenvector $\mathbf{v}_{i}^{(b)}$ of $\mathbf{W}_{i}^{(b)}$ is the optimal solution to the original problem. Otherwise, as explained before randomization procedure is proposed to generate a feasible but suboptimal solution [33], [34], [42].

\section{CSI Acquisition}

In this paper, we consider the IoT nodes to be low powered devices. These devices can range from smart wearables to smart home appliances, which may use bluetooth 4.0 LE/ 5.0 LE, IEEE 802.11n, IEEE 802.11ac, etc., along with other future communication technologies such as mmWave transmission.

We assume that the IoT devices are connected to a parent device (PD), such as a smartphone with high end signal processing capabilities. The PD has the knowledge of the nominal channels and the radius of uncertainty regions. We undertake a centralized approach where the PD collects all channel matrices, computes the beamforming matrices based on the imperfect CSI, and then distributes them to the IoT nodes.
The estimation of CSI matrices follows a similar strategy to that of traditional systems, as the IoT nodes cooperate with the PD. This is performed via the exchange of the training sequences and feedback, and the application of usual CSI estimation methods [43].

Accordingly, from a practical implementation perspective, it is important to note the following points:

- The devices considered for EH in this paper are mainly indoor devices, where the variation in channel is minimum. So once acquired, the channel can be estimated later with minimum error and less complicated signal processing.

- Devices such as smart watches, heart rate monitors, fitness trackers, etc., are usually required to be connected to a parent smart phone through bluetooth LE. These low powered devices are not required to do any complex processing to acquire the CSI, which will be the task of the more capable smart phones. Once acquired, the smart phones can transmit the information to the wearable devices.

- Devices such as Nest thermostat, Google home, Microsoft Hololens, Amazon Echo, Echo Show, etc., are usually connected to a central WiFi device. Centralized algorithms can be processed at the central device, which 
TABLE I

TOTAL PORTION OF POWER DEDICATED TO EH CONSIDERING DIFFERENT EH THRESHOLDS WHEN $K=2$.

\begin{tabular}{|c||c|c|c|c|}
\hline & $\tau=0.1$ & $\tau=0.4$ & $\tau=0.7$ & $\tau=1.0$ \\
\hline \hline$\delta=-5 \mathrm{dBm}$ & 0.6874 & 0.8687 & 0.8831 & 0.8872 \\
\hline$\delta=0 \mathrm{dBm}$ & 0.7568 & 0.8953 & 0.9348 & 0.941 \\
\hline$\delta=5 \mathrm{dBm}$ & 0.7986 & 0.9646 & 0.9394 & 0.966 \\
\hline \hline & $\tau=1.3$ & $\tau=1.6$ & $\tau=1.9$ & \\
\hline \hline$\delta=-5 \mathrm{dBm}$ & 0.8761 & 0.8949 & 0.885 & \\
\hline$\delta=0 \mathrm{dBm}$ & 0.9243 & 0.9355 & 0.9306 & \\
\hline$\delta=5 \mathrm{dBm}$ & 0.966 & 0.9518 & 0.9625 & \\
\hline
\end{tabular}

will aggregate all CSI and perform the optimization. This would however incur heavy signaling overhead and limit the network scalability, which is not an issue here as scalability is not a factor in an indoor home network.

\section{NUMERICAL RESUlTS}

In this section, we numerically investigate the SWIPT optimization problem for FD MISO and MIMO interference channels involving IoT nodes as a function of SINR constraints and inter-user interference power. Accordingly, we set the number of transmit and receive antennas at each node as $N_{i}=3$ and $M_{i}=2, i=1, \ldots, K$. For simplicity, the EH and SINR thresholds are assumed to be equal ${ }^{5}$ for all receivers, i.e., $\delta=\delta_{i}^{(a)}, \gamma=\gamma_{i}^{(a)}, \forall(i, a)$, respectively. Unless otherwise stated, the parameters used for the simulation are: $\kappa=\beta=-40 \mathrm{~dB}, \xi_{i}^{(a)}=0.5, \sigma_{i_{(a)}}^{2}=-70 \mathrm{dBm}, \forall(i, a)$, and $\sigma_{n}^{2}=-50 \mathrm{dBm}$. Iteration method's performance may rely on the initialization state. As a result, it is important to select good initialization points to achieve a suboptimal solution with a good performance. While various initialization techniques, such as random initialization, right singular matrix initialization, etc., are used in literature [44], due to the problem complexity, in this paper we use the random intialization method, which is also very common in literature [45]. The tolerance (the difference between cost function of two iterations) of the proposed iterative algorithm is set to $10^{-5}$, the maximum number of iterations is set to 50 , and the results are averaged over 1000 independent channel realizations. The entries of the channel $\mathbf{H}_{i j}^{(a c)}, \forall(i, j, a, c)$ are i.i.d. zero-mean with variance $\sigma_{i j, a c}^{2}$. For the direct channels, i.e., $\mathbf{H}_{i i}^{(a b)}, i=1, \ldots, K, a \neq b, \sigma_{i i}^{a b^{2}}=10^{-4}$. For the interuser interference channels, i.e., $\mathbf{H}_{i j}^{(a c)}, i \neq j, \sigma_{i j}^{a c 2}=\frac{10^{-4}}{\tau}$, with $\tau$ being the inter-user interference suppression factor and for the self-interference channel $\mathbf{H}_{i i}^{(a a)}, \forall(i, a), \sigma_{i i}^{a a 2}=10^{-3}$.

We begin by showing the evolution of the proposed algorithm, i.e., its convergence in Fig. 2. Here, the SINR threshold $=20 \mathrm{~dB}$. The monotonic decrease of the cost function (sum power in $\mathrm{dBm}$ ) can be verified from the figure.

After establishing the convergence of the proposed algorithm, we now show the amount of power dedicated to $\mathrm{EH}$ with different $\mathrm{EH}$ thresholds in Table I when $K=2$ and the

\footnotetext{
${ }^{5}$ In practice however, thresholds for devices will depend on their transmission power and battery capacity. For example, small devices such as wearables, might only be interested in EH, while larger devices such as smart speakers may perform EH while also transmit substantial energy for others to harvest without its quality of service (QoS) being affected.
}

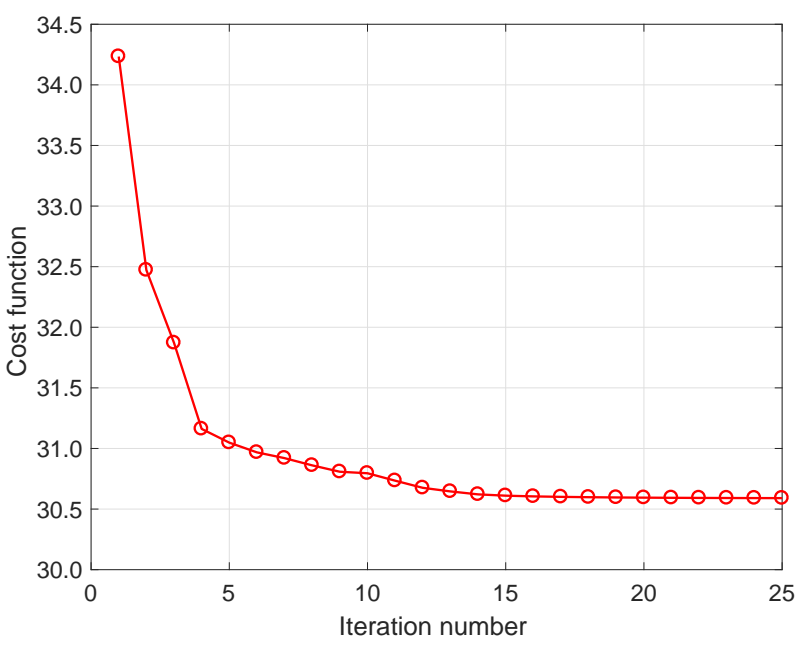

Fig. 2. Convergence of the proposed algorithm

TABLE II

PORTION OF POWER AT EACH NODE DEDICATED TO EH WHEN $\gamma_{i}^{(a)}=10 \mathrm{~dB}$ AND $\delta_{i}^{(a)}=0 \mathrm{dBm}$.

\begin{tabular}{|c|c|c||c|c|c|}
\hline$\tau=0.1$ & $a=1$ & $a=2$ & $\tau=0.7$ & $a=1$ & $a=2$ \\
\hline$K=1$ & 0.0569 & 0.0560 & $K=1$ & 0.0141 & 0.0155 \\
\hline$K=2$ & 0.0758 & 0.0545 & $K=2$ & 0.0155 & 0.0201 \\
\hline \hline$\tau=1.3$ & $a=1$ & $a=2$ & $\tau=1.9$ & $a=1$ & $a=2$ \\
\hline$K=1$ & 0.0222 & 0.0204 & $K=1$ & 0.0166 & 0.0206 \\
\hline$K=2$ & 0.0183 & 0.0148 & $K=2$ & 0.0164 & 0.0158 \\
\hline
\end{tabular}

SINR threshold $\gamma_{i}^{(a)}=10 \mathrm{~dB}$. It is worth noting that the values in this table are the portions of total power dedicated to $\mathrm{EH}$ in the system. The portions increase when the EH threshold increases which is quite intuitive. However, the variance of inter-user interference channel decreases with the increase in $\tau$. However, no linear relation can be explicitly seen between the values of $\tau$ and the portions of power. Nonetheless, it can be seen that achieved power portion values are minimum when $\tau=0.1$. In other words, when the inter-user interference is more, the amount of dedicated power required for $\mathrm{EH}$ is less. This result clearly shows the usefulness of inter-user interference in $\mathrm{EH}$ arising due to operating in FD mode. Furthermore, the optimal portion of power for each node has also been derived. For example, the optimal portion of $\mathrm{EH}$ for each node when $\gamma_{i}^{(a)}=10 \mathrm{~dB}$ and $\delta_{i}^{(a)}=0 \mathrm{dBm}$ is shown in Table II.

Next, the minimum power consumption of FD MIMO interference channel with different $\mathrm{EH}$ and SINR thresholds is shown in Fig. 3 when $K=2$. It can be observed that the system power consumption increases for higher EH threshold requirements. Thus, more power is required as SINR threshold increases. For a specific EH threshold, the power consumption increases slowly with the SINR threshold. This can be attributed to the fact that the system requires more power for signal detection.

In Fig. 4, the importance of smart channel assignment, at a stage prior to the precoder/decoder design is depicted for the proposed algorithm. The value of $\tau$ represents the provided isolation among the nodes responsible for inter user 


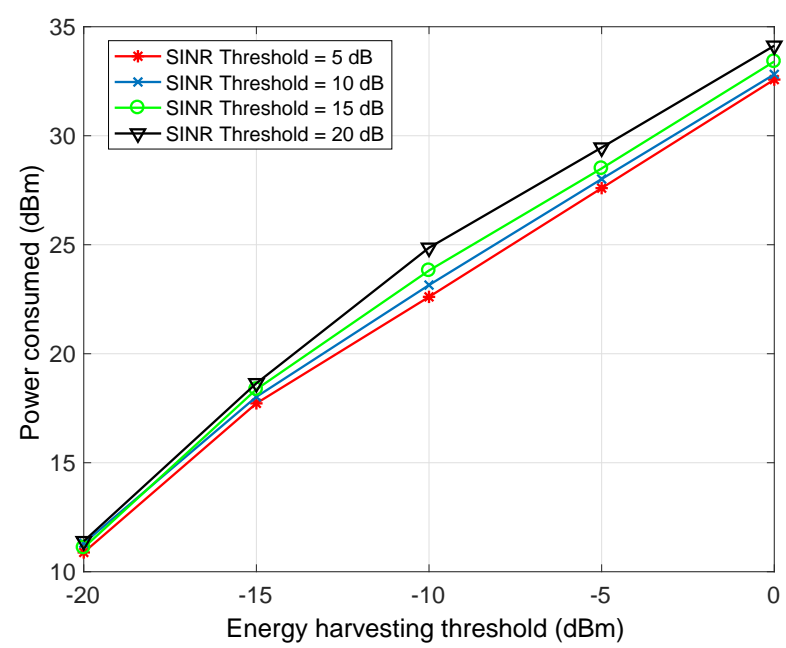

Fig. 3. Power consumption of FD MIMO interference channel with different $\mathrm{EH}$ and SINR thresholds.

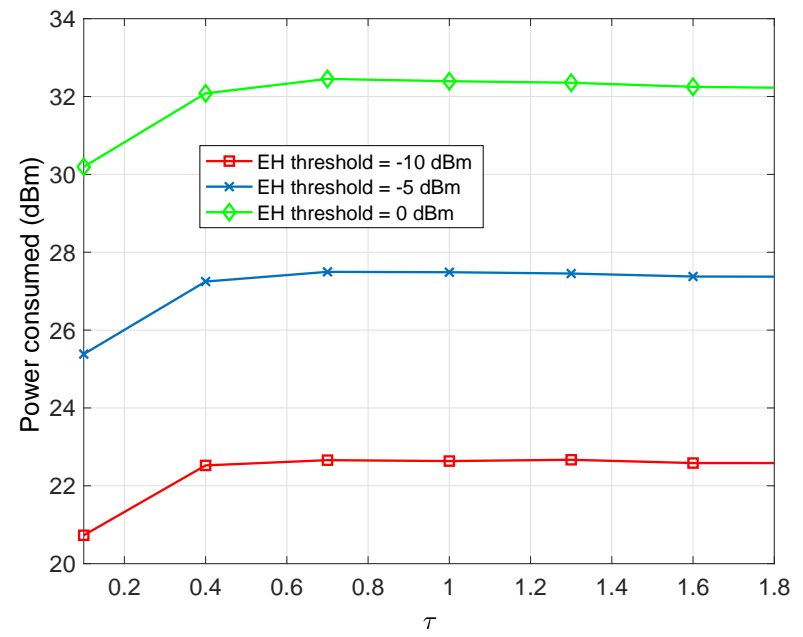

Fig. 4. Power consumption of FD MIMO interference channel for different values of $\tau$ and $\mathrm{EH}$ thresholds.

interference. In particular, the power consumption of FD MIMO interference channel with respect to the different values of $\tau$ and EH threshold when $K=2$ is presented. The SINR threshold is fixed in this figure at $10 \mathrm{~dB}$. It can be seen that the power consumption increases with $\tau$, but becomes flat later. It is worth noting that the inter-user interferences are stronger when $\tau$ is small and the system can harvest more energy from the interferences. However, the system doesn't harvest enough energy from the interference channels when $\tau$ increases. Like before, this figure also illustrates the benefits of inter-user interference in $\mathrm{EH}$.

In Fig. 5, we give the simulations for the MISO scenario. Assuming SINR threshold to be $20 \mathrm{~dB}$, we compare the performance of average normalized total transmitted power versus EH threshold for the case of 2,4 and 6 nodes when $\tau=1$. It can be seen that the average value of the total transmitted power increases monotonically with the increase of the EH threshold. Furthermore, with the increase in the

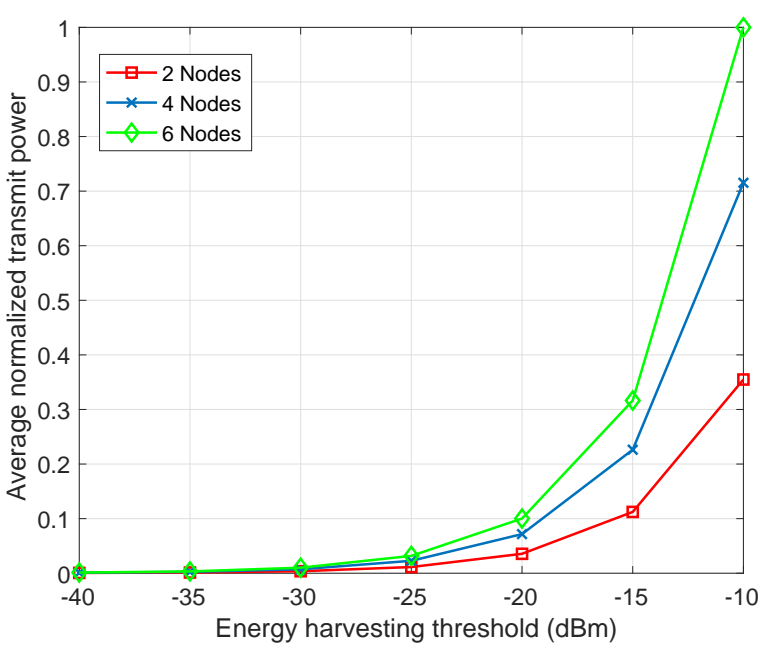

Fig. 5. Average normalized total transmitted power versus EH threshold of FD MISO interference channel.

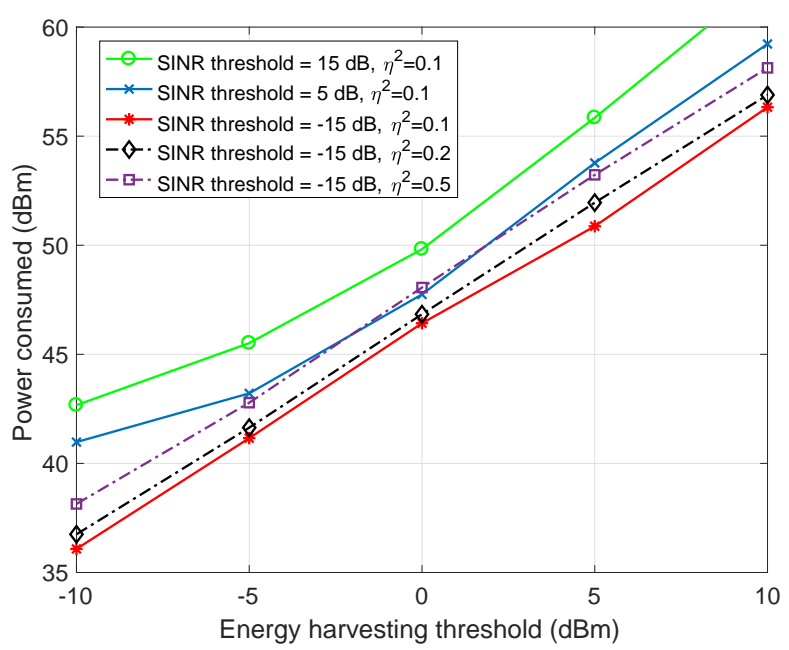

Fig. 6. Power consumption of imperfect FD MIMO interference channel for different SINR thresholds and error bounds.

number of nodes, the average normalized transmitted power also increases. It is worthwhile to note that the average normalized power increases slightly when EH threshold is less than $-15 \mathrm{~dB}$. It means that the EH technique can be applied when the required energy is small, which was also applicable for the MIMO case.

Finally, Fig. 6 and 7 present the total system power consumption of imperfect FD MIMO IoT nodes. The numbers of transmit and receive antennas at each node are set as $N_{i}=2$ and $M_{i}=2, i=1, \ldots, K$ and $K=2$. In Fig. 6, the power consumption is much higher than the perfect CSI scenario and the difference in power consumption for different SINR thresholds are more obvious. Meanwhile, it shows that more power is needed when the EH threshold increases. Further, the bound of CSI error is verified in Fig. 6 from $\eta=\sqrt{0.1}$ to $\eta=\sqrt{0.5}$. As expected, the power consumption increases with the increase in channel uncertainty. Finally, the total power consumption is plotted with respect to $\tau$ and 


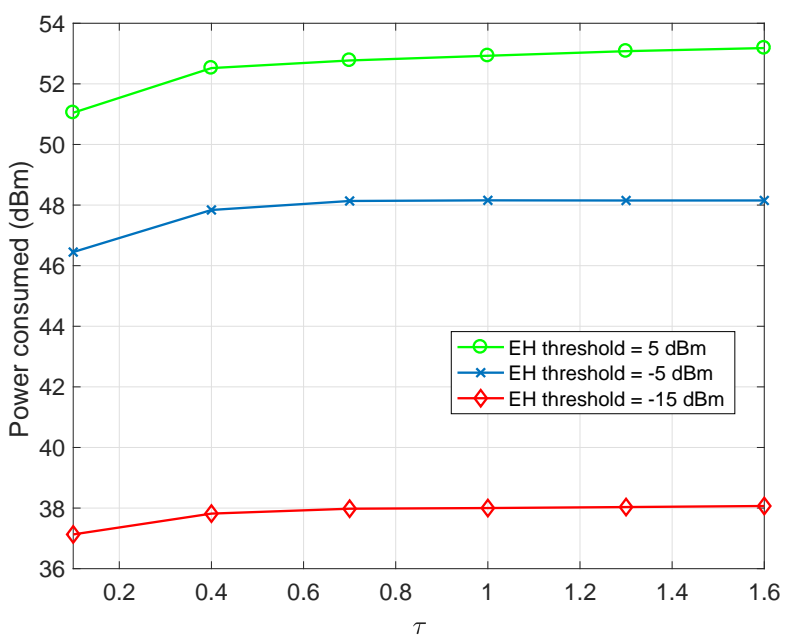

Fig. 7. Power consumption of imperfect FD MIMO interference channel for different values of $\tau$ and $\mathrm{EH}$ thresholds.

different EH thresholds in Fig. 7, where the SINR threshold is $10 \mathrm{~dB}$ and the CSI error is bounded by $\eta=\sqrt{0.1}$. Similar to the perfect CSI scenario, the power consumption increases with $\tau$, but becomes flat later. More power is needed owing to the uncertainty of the channel, but it also shows that the EH process is the primary beneficiary from the inter-user interference.

At this point it is worthwhile to mention that the $\mathrm{EH}$ threshold value chosen for the numerical analysis, satisfies the power consumption requirement of small devices, such as small low power IoT devices. Accordingly, the harvested power can definitely be considered to be utile if not sufficient to recharge their individual batteries.

\section{CONCLUding REMARKS}

The joint transceiver design problem for the SWIPT FD MISO/MIMO interference channels involving IoT nodes was studied under both perfect and imperfect CSI cases. An iterative alternating algorithm was proposed to design the transmit and receive filters. Meanwhile, the optimal power splitting ratio minimizing the total transmit power was derived.

The simulation results show that the EH technique works efficiently on FD MIMO IoT systems and the harvested power from EH can support charging of batteries of power consumption limited IoT devices with a specific guaranteed transmission quality.

Furthermore, in the current work for the sake of simplicity, we do not consider the dynamics of the battery to be charged. Several discretization techniques can be used to approximate the battery level after storing the harvested energy. In future, we will use steady-state probability analysis to derive the probability of having sufficient energy to charge the battery.

Also, we consider linear EH circuits in this work for analytical simplicity. While in linear EH model, the RF-todirect current (DC) power conversion efficiency is independent of the input impedence of the EH circuit, in a non-linear endto-end wireless power transfer circuit, this is not the case. The non-linear EH model will ensure better resource allocation for SWIPT, which will be considered in future as an extension to this work.

\section{APPENDIX A \\ PROBlem REFORMULATION (IMPERFECT CSI-MISO CASE)}

Here, we show the steps for reformulating the problem (26). To begin, for the SINR constraint, we introduce three auxiliary variables as

$$
\begin{aligned}
a_{i i}^{a b}= & \max _{\forall \mathbf{e}_{i i}^{a b} \mathbf{e}_{i i}^{a b} \leq \eta_{i i}^{a b}}\left(\mathbf{h}_{i i}^{(a b)}\right)^{H}(\kappa(1+\beta) \\
& \left.\times \operatorname{diag}\left(\mathbf{v}_{i}^{(b)}\left(\mathbf{v}_{i}^{(b)}\right)^{H}\right)+\beta \mathbf{v}_{i}^{(b)}\left(\mathbf{v}_{i}^{(b)}\right)^{H}\right)\left(\mathbf{h}_{i i}^{(a b)}\right), \\
b_{i i}^{a a}= & \max _{\forall \mathbf{e}_{i i}^{a a} \mathbf{e}_{i i}^{a a} \leq \eta_{i i}^{a a 2}}\left(\mathbf{h}_{i i}^{(a a)}\right)^{H}(\kappa(1+\beta) \\
& \left.\times \operatorname{diag}\left(\mathbf{v}_{i}^{(a)}\left(\mathbf{v}_{i}^{(a)}\right)^{H}\right)+\beta \mathbf{v}_{i}^{(a)}\left(\mathbf{v}_{i}^{(a)}\right)^{H}\right)\left(\mathbf{h}_{i i}^{(a a)}\right), \\
& c_{i j}^{a c}=\max _{\forall \mathbf{e}_{i j}^{a c H} \mathbf{e}_{i j}^{a c} \leq \eta_{i j}^{a c 2}}\left(\mathbf{h}_{i j}^{(a c)}\right)^{H}\left(\mathbf{v}_{j}^{(c)}\left(\mathbf{v}_{j}^{(c)}\right)^{H}\right. \\
& \left.+\kappa \operatorname{diag}\left(\mathbf{v}_{j}^{(c)}\left(\mathbf{v}_{j}^{(c)}\right)^{H}\right)\right)\left(\mathbf{h}_{i j}^{(a c)}\right),
\end{aligned}
$$

where $a_{i i}^{a b}, b_{i i}^{a a}$ and $c_{i j}^{a c}$ are the maximum (worst cases) cross tier and self-interference. By using these three auxiliary variables, $\Sigma_{i}^{(a)}$ in eq. (7) can be rewritten as

$$
\bar{\Sigma}_{i}^{(a)}=a_{i i}^{a b}+b_{i i}^{a a}+(1+\beta)\left(\sum_{j \neq i}^{K} \sum_{c=1}^{2} c_{i j}^{a c}+\sigma_{n}^{2}\right) .
$$

Then, the SINR constraint can be rewritten as

$$
\begin{aligned}
& \rho_{i}^{(a)}\left(\widetilde{\mathbf{h}}_{i i}^{(a b)}+\mathbf{e}_{i i}^{a b}\right)^{H} \mathbf{W}_{i}^{(b)}\left(\widetilde{\mathbf{h}}_{i i}^{(a b)}+\mathbf{e}_{i i}^{a b}\right) \\
& -\gamma_{i}^{(a)} \rho_{i}^{(a)} \bar{\Sigma}_{i}^{(a)}-\gamma_{i}^{(a)} \sigma_{i(a)}^{2} \geq 0,\left\|\mathbf{e}_{i i}^{a b}\right\|^{2} \leq \eta_{i i}^{a b^{2}} \\
& a_{i i}^{a b} \geq\left(\widetilde{\mathbf{h}}_{i i}^{(a b)}+\mathbf{e}_{i i}^{a b}\right)^{H}\left(\kappa(1+\beta) \operatorname{diag}\left(\mathbf{W}_{i}^{(b)}\right)+\beta \mathbf{W}_{i}^{(b)}\right) \\
& \times\left(\widetilde{\mathbf{h}}_{i i}^{(a b)}+\mathbf{e}_{i i}^{a b}\right),\left\|\mathbf{e}_{i i}^{a b}\right\|^{2} \leq \eta_{i i}^{a b^{2}}, \\
& b_{i i}^{a a} \geq\left(\widetilde{\mathbf{h}}_{i i}^{(a a)}+\mathbf{e}_{i i}^{a a}\right)^{H}\left(\kappa(1+\beta) \operatorname{diag}\left(\mathbf{W}_{i}^{(a)}\right)+\beta \mathbf{W}_{i}^{(a)}\right) \\
& \times\left(\widetilde{\mathbf{h}}_{i i}^{(a a)}+\mathbf{e}_{i i}^{a a}\right),\left\|\mathbf{e}_{i i}^{a a}\right\|^{2} \leq \eta_{i i}^{a a 2}, \\
& c_{i j}^{a c} \geq\left(\widetilde{\mathbf{h}}_{i j}^{(a c)}+\mathbf{e}_{i j}^{a c}\right)^{H}\left(\mathbf{W}_{j}^{(c)}+\kappa \operatorname{diag}\left(\mathbf{W}_{j}^{(c)}\right)\right) \\
& \times\left(\widetilde{\mathbf{h}}_{i j}^{(a c)}+\mathbf{e}_{i j}^{a c}\right),\left\|\mathbf{e}_{i j}^{a c}\right\|^{2} \leq \eta_{i j}^{a c 2},
\end{aligned}
$$

for $(i, j) \in\{1, \ldots, K\}$ and $(a, b) \in\{1,2\}$.

Similarly, for EH constraint, we introduce four auxiliary variables as (40), (41), (42) and (43), where $\widehat{a}_{i i}^{a b}, \widehat{b}_{i i}^{a a}, \widehat{c}_{i j}^{a c}$ and $d_{i i}^{a a}$ denote the minimum (worst cases) available power for 


$$
\begin{aligned}
& \widehat{a}_{i i}^{a b}=\min _{\forall \mathbf{e}_{i i}^{a b} \mathbf{e}_{i i}^{a b} \leq \eta_{i i}^{a b}}\left(\mathbf{h}_{i i}^{(a b)}\right)^{H}\left(\kappa(1+\beta) \operatorname{diag}\left(\mathbf{v}_{i}^{(b)}\left(\mathbf{v}_{i}^{(b)}\right)^{H}\right)+\beta \mathbf{v}_{i}^{(b)}\left(\mathbf{v}_{i}^{(b)}\right)^{H}\right)\left(\mathbf{h}_{i i}^{(a b)}\right), \\
& \widehat{b}_{i i}^{a a}=\min _{\forall \mathbf{e}_{i i}^{a a} \mathbf{e}_{i i}^{a a} \leq \eta_{i i}^{a a 2}}\left(\mathbf{h}_{i i}^{(a a)}\right)^{H}\left(\kappa(1+\beta) \operatorname{diag}\left(\mathbf{v}_{i}^{(a)}\left(\mathbf{v}_{i}^{(a)}\right)^{H}\right)+\beta \mathbf{v}_{i}^{(a)}\left(\mathbf{v}_{i}^{(a)}\right)^{H}\right)\left(\mathbf{h}_{i i}^{(a a)}\right), \\
& \widehat{c}_{i j}^{a c}=\min _{\forall \mathbf{e}_{i j}^{a c} \mathbf{e}_{i j}^{a c} \leq \eta_{i j}^{a c 2}}\left(\mathbf{h}_{i j}^{(a c)}\right)^{H}\left(\mathbf{v}_{j}^{(c)}\left(\mathbf{v}_{j}^{(c)}\right)^{H}+\kappa \operatorname{diag}\left(\mathbf{v}_{j}^{(c)}\left(\mathbf{v}_{j}^{(c)}\right)^{H}\right)\right)\left(\mathbf{h}_{i j}^{(a c)}\right), \\
& d_{i i}^{a a}=\min _{\forall \mathbf{e}_{i i}^{a{ }^{a} H} \mathbf{e}_{i i}^{a a} \leq \eta_{i i}^{a a 2}}\left(\mathbf{h}_{i i}^{(a a)}\right)^{H}\left(\mathbf{v}_{i}^{(a)}\left(\mathbf{v}_{i}^{(a)}\right)^{H}\right)\left(\mathbf{h}_{i i}^{(a a)}\right),
\end{aligned}
$$

EH from all the nodes. The covariance matrix in EH can be rewritten as

$$
\bar{\Sigma}_{i}^{(a)}=\widehat{a}_{i i}^{a b}+\widehat{b}_{i i}^{a a}+(1+\beta)\left(\sum_{j \neq i}^{K} \sum_{c=1}^{2} \widehat{c}_{i j}^{a c}+\sigma_{n}^{2}\right) .
$$

Then, the EH constraint can be rewritten as

$$
\begin{aligned}
& \xi_{i}^{(a)}\left(1-\rho_{i}^{(a)}\right)\left(\left(\widetilde{\mathbf{h}}_{i i}^{(a b)}+\mathbf{e}_{i i}^{a b}\right)^{H} \mathbf{W}_{i}^{(b)}\left(\widetilde{\mathbf{h}}_{i i}^{(a b)}+\mathbf{e}_{i i}^{a b}\right)\right. \\
& \left.+d_{i i}^{a a}+\widehat{\Sigma}_{i}^{(a)}\right)-\delta_{i}^{(a)} \geq 0,\left\|\mathbf{e}_{i i}^{a b}\right\|^{2} \leq \eta_{i i}^{a b^{2}} \\
& \widehat{a}_{i i}^{a b} \leq\left(\widetilde{\mathbf{h}}_{i i}^{(a b)}+\mathbf{e}_{i i}^{a b}\right)^{H}\left(\kappa(1+\beta) \operatorname{diag}\left(\mathbf{W}_{i}^{(b)}\right)\right. \\
& \left.+\beta \mathbf{W}_{i}^{(b)}\right)\left(\widetilde{\mathbf{h}}_{i i}^{(a b)}+\mathbf{e}_{i i}^{a b}\right),\left\|\mathbf{e}_{i i}^{a b}\right\|^{2} \leq \eta_{i i}^{a b^{2}} \\
& \widehat{b}_{i i}^{a a} \leq\left(\widetilde{\mathbf{h}}_{i i}^{(a a)}+\mathbf{e}_{i i}^{a a}\right)^{H}\left(\kappa(1+\beta) \operatorname{diag}\left(\mathbf{W}_{i}^{(a)}\right)\right. \\
& \left.+\beta \mathbf{W}_{i}^{(a)}\right)\left(\widetilde{\mathbf{h}}_{i i}^{(a a)}+\mathbf{e}_{i i}^{a a}\right),\left\|\mathbf{e}_{i i}^{a a}\right\|^{2} \leq \eta_{i i}^{a a 2} \\
& \quad \widehat{c}_{i j}^{a c} \leq\left(\widetilde{\mathbf{h}}_{i j}^{(a c)}+\mathbf{e}_{i j}^{a c}\right){ }^{H}\left(\mathbf{W}_{j}^{(c)}+\kappa \operatorname{diag}\left(\mathbf{W}_{j}^{(c)}\right)\right) \\
& \quad \times\left(\widetilde{\mathbf{h}}_{i j}^{(a c)}+\mathbf{e}_{i j}^{a c}\right),\left\|\mathbf{e}_{i j}^{a c}\right\|^{2} \leq \eta_{i j}^{a c 2} \\
& d_{i i}^{a a} \leq\left(\widetilde{\mathbf{h}}_{i i}^{(a a)}+\mathbf{e}_{i i}^{a a}\right)^{H}\left(\mathbf{W}_{i}^{(a)}\right) \\
& \quad \times\left(\widetilde{\mathbf{h}}_{i i}^{(a a)}+\mathbf{e}_{i i}^{a a}\right),\left\|\mathbf{e}_{i i}^{a a}\right\|^{2} \leq \eta_{i i}^{a a 2} \\
& \quad \text { for }(i, j) \in\{1, \ldots, K\} \text { and }(a, b) \in\{1,2\}
\end{aligned}
$$

However, this problem is still computationally intractable, because it involves an infinite number of constraints, which have to be reformulated into finite convex constraints. By applying the S-procedure [39], the SINR constraints in (36), (37), (38) and (39) can be reformulated to finite convex constraints which are equivalent to (50), (51), (52) and (53), where

$$
\begin{aligned}
& \mathbf{F}_{1}=\kappa(1+\beta) \operatorname{diag}\left(\mathbf{W}_{i}^{(b)}\right)+\beta \mathbf{W}_{i}^{(b)}, \\
& \mathbf{F}_{2}=\kappa(1+\beta) \operatorname{diag}\left(\mathbf{W}_{i}^{(a)}\right)+\beta \mathbf{W}_{i}^{(a)}, \\
& \mathbf{F}_{3}=\mathbf{W}_{j}^{(c)}+\kappa \operatorname{diag}\left(\mathbf{W}_{j}^{(c)}\right) .
\end{aligned}
$$

Meanwhile, the EH constraints in (45), (46), (47), (48) and (49) can be reformulated to finite convex constraints which are equivalent to (57), (58), (59), (60) and (61).

Accordingly, after some simple mathematical manipulations, we obtain (28).

\section{APPENDIX B \\ Problem ReFormulation (IMPERFECT CSI-MIMO CASE)}

Here, we show the steps for reformulating the problem (31). To begin, the SINR constraint can be rewritten as (62).

Then, the SINR constraint of this MIMO scenario can be reformulated as (63), (64), (65), (66), (67) and (68), where

$$
\begin{aligned}
\bar{\Sigma}_{i}^{(a)} \triangleq & \kappa\left(a_{i i}^{a b}+b_{i i}^{a a}\right)+\sum_{j \neq i}^{K} \sum_{c=1}^{2} c_{i j}^{a c}+\sum_{j=1}^{K} \sum_{c=1}^{2} \beta d_{i j}^{a c} \\
& +\left(\mathbf{u}_{i}^{a}\right)^{H} \sigma_{n}^{2} \mathbf{I} \mathbf{u}_{i}^{a},
\end{aligned}
$$

$$
\begin{gathered}
V S 1 \triangleq \min _{\left\|\mathbf{E}_{i j}^{(a b)}\right\|_{F} \leq \eta_{i j}^{(a b)}} \frac{1}{\gamma_{i}^{(a)}}\left(\left(\mathbf{u}_{i}^{(a)}\right)^{H}\left(\tilde{\mathbf{H}}_{i i}^{(a b)}+\mathbf{E}_{i i}^{(a b)}\right)\right. \\
\left.\times \mathbf{W}_{i}^{(b)}\left(\tilde{\mathbf{H}}_{i i}^{(a b)}+\mathbf{E}_{i i}^{(a b)}\right)^{H} \mathbf{u}_{i}^{(a)}\right) \\
\mathbf{F}_{4}=\left(\mathbf{W}_{j}^{(c)}+\kappa \operatorname{diag}\left(\mathbf{W}_{j}^{(c)}\right)\right)
\end{gathered}
$$$$
a_{i i}^{a b} \triangleq \max _{\forall\left\|\mathbf{E}_{i i}^{a b}\right\|_{F}^{2} \leq \eta_{i i}^{a b} 2}\left(\mathbf{u}_{i}^{a}\right)^{H} \mathbf{H}_{i i}^{(a b)} \operatorname{diag}\left(\mathbf{W}_{i}^{(b)}\right)\left(\mathbf{H}_{i i}^{(a b)}\right)^{H} \mathbf{u}_{i}^{a},
$$

$$
b_{i i}^{a a} \triangleq \max _{\forall\left\|\mathbf{E}_{i i}^{a a}\right\|_{F}^{2} \leq \eta_{i i}^{a a 2}}\left(\mathbf{u}_{i}^{a}\right)^{H} \mathbf{H}_{i i}^{(a a)} \operatorname{diag}\left(\mathbf{W}_{i}^{(a)}\right)\left(\mathbf{H}_{i i}^{(a a)}\right)^{H} \mathbf{u}_{i}^{a},
$$

$$
c_{i j}^{a c} \triangleq \max _{\forall\left\|\mathbf{E}_{i j}^{a c}\right\|_{F}^{2} \leq \eta_{i j}^{a c 2}}\left(\mathbf{u}_{i}^{a}\right)^{H} \mathbf{H}_{i j}^{(a c)} \mathbf{F}_{4}\left(\mathbf{H}_{i j}^{(a c)}\right)^{H} \mathbf{u}_{i}^{a},
$$

$$
d_{i j}^{a c} \triangleq \max _{\forall\left\|\mathbf{E}_{i j}^{a c}\right\|_{F}^{2} \leq \eta_{i j}^{a c 2}}\left(\mathbf{u}_{i}^{a}\right)^{H} \operatorname{diag}\left(\mathbf{H}_{i j}^{(a c)} \mathbf{W}_{j}^{(c)}\left(\mathbf{H}_{i j}^{(a c)}\right)^{H}\right) \mathbf{u}_{i}^{a} .
$$

and $\lambda_{i j}^{a b}$ are the slack variables. Note that, the slack variables in (64) and (65) should be marked differently for simulations, which use the same $\lambda_{i i}^{a b}$. Similarly, the EH constraint can be reformulated as (73), (74), (75), (76), (77), (78) and (79), where 


$$
\begin{gathered}
\left(\begin{array}{cc}
\mathbf{W}_{i}^{(b)}+\lambda_{i i}^{a b} \mathbf{I} & \mathbf{W}_{i}^{(b)}\left(\widetilde{\mathbf{h}}_{i i}^{(a b)}\right) \\
\left(\widetilde{\mathbf{h}}_{i i}^{(a b)}\right)^{H} \mathbf{W}_{i}^{(b)} & \left(\widetilde{\mathbf{h}}_{i i}^{(a b)}\right)^{H} \mathbf{W}_{i}^{(b)}\left(\widetilde{\mathbf{h}}_{i i}^{(a b)}\right)-\gamma_{i}^{(a)} \rho_{i}^{(a)} \bar{\Sigma}_{i}^{(a)}-\gamma_{i}^{(a)} \sigma_{i(a)}^{2}-\lambda_{i i}^{a b} \eta_{i i}^{a b^{2}}
\end{array}\right) \succeq 0, \\
\left(\begin{array}{cc}
-\mathbf{F}_{1}+\lambda_{i i}^{a b} \mathbf{I} & -\mathbf{F}_{1}\left(\widetilde{\mathbf{h}}_{i i}^{(a b)}\right) \\
-\left(\widetilde{\mathbf{h}}_{i i}^{(a b)}\right)^{H} \mathbf{F}_{1} & a_{i i}^{a b}-\left(\widetilde{\mathbf{h}}_{i i}^{(a b)}\right)^{H} \mathbf{F}_{1}\left(\widetilde{\mathbf{h}}_{i i}^{(a b)}\right)-\lambda_{i i}^{a b} \eta_{i i}^{a b}
\end{array}\right) \succeq 0, \\
\left(\begin{array}{cc}
-\mathbf{F}_{2}+\lambda_{i i}^{a a} \mathbf{I} & -\mathbf{F}_{2}\left(\widetilde{\mathbf{h}}_{i i}^{(a a)}\right) \\
-\left(\widetilde{\mathbf{h}}_{i i}^{(a a)}\right)^{H} \mathbf{F}_{2} & b_{i i}^{a a}-\left(\widetilde{\mathbf{h}}_{i i}^{(a a)}\right)^{H} \mathbf{F}_{2}\left(\widetilde{\mathbf{h}}_{i i}^{(a a)}\right)-\lambda_{i i}^{a a} \eta_{i i}^{a a 2}
\end{array}\right) \succeq 0, \\
\left(\begin{array}{cc}
-\mathbf{F}_{3}+\lambda_{i j}^{a c} \mathbf{I} & -\mathbf{F}_{3}\left(\widetilde{\mathbf{h}}_{i j}^{(a c)}\right), \\
-\left(\widetilde{\mathbf{h}}_{i j}^{(a c)}\right)^{H} \mathbf{F}_{3} & c_{i j}^{a c}-\left(\widetilde{\mathbf{h}}_{i j}^{(a c)}\right)^{H} \mathbf{F}_{3}\left(\widetilde{\mathbf{h}}_{i j}^{(a c)}\right)-\lambda_{i j}^{a c} \eta_{i j}^{a c 2}
\end{array}\right) \succeq 0,
\end{gathered}
$$

$$
\begin{aligned}
& \rho_{i}^{(a)} \geq \sigma_{i(a)}^{2}\left[\frac{\left(\left(\mathbf{u}_{i}^{(a)}\right)^{H}\left(\tilde{\mathbf{H}}_{i i}^{(a b)}+\mathbf{E}_{i i}^{(a b)}\right) \mathbf{W}_{i}^{(b)}\left(\tilde{\mathbf{H}}_{i i}^{(a b)}+\mathbf{E}_{i i}^{(a b)}\right)^{H} \mathbf{u}_{i}^{(a)}\right)-\left(\mathbf{u}_{i}^{(a)}\right)^{H} \hat{\mathbf{\Sigma}}_{i}^{(a)} \mathbf{u}_{i}^{(a)}}{\gamma_{i}^{(a)}}\right]^{-1} \\
& \Rightarrow\left(\begin{array}{cc}
\rho_{i}^{(a)} & \sigma_{i(a)} \\
\sigma_{i(a)} & \frac{\left(\left(\mathbf{u}_{i}^{(a)}\right)^{H}\left(\tilde{\mathbf{H}}_{i i}^{(a b)}+\mathbf{E}_{i i}^{(a b)}\right) \mathbf{W}_{i}^{(b)}\left(\tilde{\mathbf{H}}_{i i}^{(a b)}+\mathbf{E}_{i i}^{(a b)}\right)^{H} \mathbf{u}_{i}^{(a)}\right)-\left(\mathbf{u}_{i}^{(a)}\right)^{H} \overline{\mathbf{\Sigma}}_{i}^{(a)} \mathbf{u}_{i}^{(a)}}{\gamma_{i}^{(a)}}
\end{array}\right) \succeq 0 .
\end{aligned}
$$

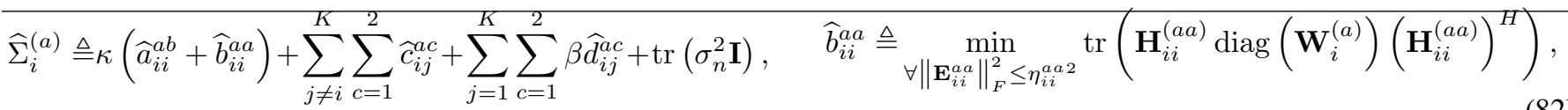

$$
\begin{aligned}
& V S 2 \triangleq \min _{\left\|\mathbf{E}_{i j}^{(a b)}\right\|_{F} \leq \eta_{i j}^{(a b)}} \operatorname{tr}\left\{\left(\tilde{\mathbf{H}}_{i i}^{(a b)}+\mathbf{E}_{i i}^{(a b)}\right)\right. \\
& \left.\times \mathbf{W}_{i}^{(b)}\left(\mathbf{H}_{i i}^{(a b)}+\mathbf{E}_{i i}^{(a b)}\right)^{H}\right\}, \\
& \bar{a}_{i i}^{a a} \triangleq \min _{\forall\left\|\mathbf{E}_{i i}^{a a}\right\|_{F}^{2} \leq \eta_{i i}^{a a} 2} \operatorname{tr}\left(\mathbf{H}_{i i}^{(a a)} \mathbf{W}_{i}^{(a)}\left(\mathbf{H}_{i i}^{(a a)}\right)^{H}\right), \\
& \widehat{c}_{i j}^{a c} \triangleq \min _{\forall\left\|\mathbf{E}_{i j}^{a c}\right\|_{F}^{2} \leq \eta_{i j}^{a c 2}} \operatorname{tr}\left(\mathbf{H}_{i j}^{(a c)} \mathbf{F}_{4}\left(\mathbf{H}_{i j}^{(a c)}\right)^{H}\right), \\
& \widehat{a}_{i i}^{a b} \triangleq \min _{\forall\left\|\mathbf{E}_{i i}^{a b}\right\|_{F}^{2} \leq \eta_{i i}^{a b 2}} \operatorname{tr}\left(\mathbf{H}_{i i}^{(a b)} \operatorname{diag}\left(\mathbf{W}_{i}^{(b)}\right)\left(\mathbf{H}_{i i}^{(a b)}\right)^{H}\right), \\
& \widehat{d}_{i j}^{a c} \triangleq \min _{\forall\left\|\mathbf{E}_{i j}^{a c}\right\|_{F}^{2} \leq \eta_{i j}^{a c 2}} \operatorname{tr}\left(\operatorname{diag}\left(\mathbf{H}_{i j}^{(a c)} \mathbf{W}_{j}^{(c)}\left(\mathbf{H}_{i j}^{(a c)}\right)^{H}\right)\right) .
\end{aligned}
$$




$$
\begin{aligned}
& \left(\begin{array}{ll}
\rho_{i}^{(a)} & \sigma_{i(a)} \\
\sigma_{i(a)} & V S 1-\left(\mathbf{u}_{i}^{(a)}\right)^{H} \overline{\boldsymbol{\Sigma}}_{i}^{(a)} \mathbf{u}_{i}^{(a)}
\end{array}\right) \succeq 0, \\
& \left(\begin{array}{cc}
\frac{1}{\gamma_{i}^{(a)}}\left(\mathbf{u}_{i}^{(a)}\right)^{H} \tilde{\mathbf{H}}_{i i}^{(a b)} \mathbf{W}_{i}^{(b)}\left(\tilde{\mathbf{H}}_{i i}^{(a b)}\right)^{H} \mathbf{u}_{i}^{(a)}-V S 1-\lambda_{i i}^{a b} & \left(\mathbf{u}_{i}^{(a)}\right)^{H} \tilde{\mathbf{H}}_{i i}^{(a b)} \mathbf{W}_{i}^{(b)} \\
\left(\left(\mathbf{u}_{i}^{(a)}\right)^{H} \tilde{\mathbf{H}}_{i i}^{(a b)} \mathbf{W}_{i}^{(b)}\right)^{H} & \mathbf{W}_{i}^{(b)}+\frac{\lambda_{i j}^{a b}}{\eta_{i i}^{(a b)}}
\end{array}\right) \succeq 0, \\
& \left(\begin{array}{cr}
-\left(\mathbf{u}_{i}^{a}\right)^{H} \operatorname{diag}\left(\mathbf{W}_{i}^{(b)}\right) \mathbf{u}_{i}^{a}+\lambda_{i i}^{a b} & -\left(\mathbf{u}_{i}^{a}\right)^{H} \operatorname{diag}\left(\mathbf{W}_{i}^{(b)}\right)\left(\widetilde{\mathbf{H}}_{i i}^{(a b)}\right)^{H} \mathbf{u}_{i}^{a} \\
-\left(\mathbf{u}_{i}^{a}\right)^{H} \widetilde{\mathbf{H}}_{i i}^{(a b)} \operatorname{diag}\left(\mathbf{W}_{i}^{(b)}\right) \mathbf{u}_{i}^{a} & a_{i i}^{a b}-\left(\mathbf{u}_{i}^{a}\right)^{H} \widetilde{\mathbf{H}}_{i i}^{(a b)} \operatorname{diag}\left(\mathbf{W}_{i}^{(b)}\right)\left(\widetilde{\mathbf{H}}_{i i}^{(a b)}\right)^{H} \mathbf{u}_{i}^{a}-\lambda_{i i}^{a b} \eta_{i i}^{a b^{2}}
\end{array}\right) \succeq 0, \\
& \left(\begin{array}{cc}
-\left(\mathbf{u}_{i}^{a}\right)^{H} \operatorname{diag}\left(\mathbf{W}_{i}^{(a)}\right) \mathbf{u}_{i}^{a}+\lambda_{i i}^{a a} & -\left(\mathbf{u}_{i}^{a}\right)^{H} \operatorname{diag}\left(\mathbf{W}_{i}^{(a)}\right)\left(\widetilde{\mathbf{H}}_{i i}^{(a a)}\right)^{H} \mathbf{u}_{i}^{a} \\
-\left(\mathbf{u}_{i}^{a}\right)^{H} \widetilde{\mathbf{H}}_{i i}^{(a a)} \operatorname{diag}\left(\mathbf{W}_{i}^{(a)}\right) \mathbf{u}_{i}^{a} & b_{i i}^{a a}-\left(\mathbf{u}_{i}^{a}\right)^{H} \widetilde{\mathbf{H}}_{i i}^{(a)} \operatorname{diag}\left(\mathbf{W}_{i}^{(a)}\right)\left(\widetilde{\mathbf{H}}_{i i}^{(a a)}\right)^{H} \mathbf{u}_{i}^{a}-\lambda_{i i}^{a a} \eta_{i i}^{a a 2}
\end{array}\right) \succeq 0, \\
& \left(\begin{array}{cc}
-\left(\mathbf{u}_{i}^{a}\right)^{H} \mathbf{F}_{4} \mathbf{u}_{i}^{a}+\lambda_{i j}^{a c} & -\left(\mathbf{u}_{i}^{a}\right)^{H} \mathbf{F}_{4}\left(\widetilde{\mathbf{H}}_{i j}^{(a c)}\right)^{H} \mathbf{u}_{i}^{a} \\
-\left(\mathbf{u}_{i}^{a}\right)^{H} \widetilde{\mathbf{H}}_{i j}^{(a c)} \mathbf{F}_{4} \mathbf{u}_{i}^{a} & c_{i j}^{a c}-\left(\mathbf{u}_{i}^{a}\right)^{H} \widetilde{\mathbf{H}}_{i j}^{(a c)} \mathbf{F}_{4}\left(\widetilde{\mathbf{H}}_{i j}^{(a c)}\right)^{H} \mathbf{u}_{i}^{a}-\lambda_{i j}^{a c} \eta_{i j}^{a c 2}
\end{array}\right) \succeq 0, \\
& \left(\begin{array}{cc}
-\left(\mathbf{u}_{i}^{a}\right)^{H} \operatorname{diag}\left(\mathbf{W}_{j}^{(c)}\right) \mathbf{u}_{i}^{a}+\lambda_{i j}^{a c} & -\left(\mathbf{u}_{i}^{a}\right)^{H} \operatorname{diag}\left(\mathbf{W}_{j}^{(c)}\left(\widetilde{\mathbf{H}}_{i j}^{(a c)}\right)^{H}\right) \mathbf{u}_{i}^{a} \\
-\left(\mathbf{u}_{i}^{a}\right)^{H} \operatorname{diag}\left(\widetilde{\mathbf{H}}_{i j}^{(a c)} \mathbf{W}_{j}^{(c)}\right) \mathbf{u}_{i}^{a} & d_{i j}^{a c}-\left(\mathbf{u}_{i}^{a}\right)^{H} \operatorname{diag}\left(\widetilde{\mathbf{H}}_{i j}^{(a c)} \mathbf{W}_{j}^{(c)}\left(\widetilde{\mathbf{H}}_{i j}^{(a c)}\right)^{H}\right) \mathbf{u}_{i}^{a}-\lambda_{i j}^{a c} \eta_{i j}^{a c 2}
\end{array}\right) \succeq 0,
\end{aligned}
$$

\section{REFERENCES}

[1] A. Goldsmith, Wireless Communications. Cambridge University Press, New York, NY, USA, 2005.

[2] V. R. Cadambe and S. A. Jafar, "Degrees of freedom of wireless networks with relays, feedback, cooperation, and full duplex operation," IEEE Trans. on Inform. Theory, vol. 55, no. 5, pp. 2334-2344, May 2009.

[3] C. Zhong, H. A. Suraweera, G. Zheng, I. Krikidis, and Z. Zhang, "Wireless information and power transfer with full duplex relaying," IEEE Trans. Commun., vol. 62, pp. 3447-3461, Oct. 2014.

[4] C.-L. I, C. Rowell, S. Han, Z. Xu, G. Li, and Z. Pan, "Towards green and soft: A 5g perspective," IEEE Commun. Mag., Feb. 2014.

[5] Z. Tong, C. Russ, S. Vanka, and M. Haenggi, "Prototype of virtual full duplex via rapid on-off-division duplex," IEEE Trans. on Commun., 2015, accepted, available at https://www3.nd.edu/ mhaenggi/pubs/tcom15a.pdf.

[6] J. Lee and T. Quek, "Hybrid full-half-duplex system analysis inheterogeneous wireless networks," IEEE Trans. on Wireless Commun., vol. 14, no. 5 , pp. 2883-2895, May 2015

[7] M. Jain, J. I. Choi, T. Kim, D. Bharadia, K. Srinivasan, S. Seth, P. Levis, S. Katti, and P. Sinha, "Practical, real-time, full duplex wireless," Proc. Mobicom, pp. 301-312, Sep. 2011

[8] M. Duarte, C. Dick, and A. Sabharwal, "Experiment-driven characterization of full-duplex wireless systems," IEEE Trans. Wireless Commun., vol. 11, no. 12, pp. 4296-4307, Dec. 2012.

[9] D. Bharadia and S. Katti, "Full duplex MIMO radios," USENIX NSDI, pp. 359-372, 2014.

[10] Y. Hua, Y. Ma, A. Gholian, Y. Li, A. C. Cirik, and P. Liang, "Radio self-interference cancellation by transmit beamforming, all-analog cancellation and blind digital tuning," Elsevier Signal Process., vol. 108, pp. 322-340, Mar. 2015.

[11] A. Sahai, G. Patel, C. Dick, and A. Sabharwal, "On the impact of phase noise on active cancelation in wireless full-duplex," EEE Trans. Vel. Technol., vol. 62, no. 9, pp. 4494-4510, Nov. 2013.

[12] B. P. Day, A. R. Margetts, D. W. Bliss, and P. Schniter, "Full-duplex bidirectional MIMO: Achievable rates under limited dynamic range," IEEE Trans. Signal Process., vol. 60, no. 7, pp. 3702-3713, Aug. 2012

[13] A. C. Cirik, Y. Rong, and Y. Hua, "Achievable rates of full-duplex MIMO radios in fast fading channels with imperfect channel estimation," IEEE Trans. Signal Processing, vol. 62, pp. 3874-3886, Aug. 2014.
[14] W. Li, J. Lilleberg, and K. Rikkinen, "On rate region analysis of half- and full-duplex OFDM communication links," IEEE J. Sel. Areas Commun., vol. 32, no. 9, pp. 1688-1698, Sep. 2014.

[15] A. C. Cirik, R. Wang, Y. Hua, and M. Latva-aho, "Weighted sum-rate maximization for full-duplex MIMO interference channels," IEEE Trans. Commun., vol. 63, no. 3, pp. 801-815, Mar. 2015.

[16] A. C. Cirik, R. Wang, Y. Rong, and Y. Hua, "MSE-based transceiver designs for full-duplex MIMO cognitive radios," IEEE Trans. Commun., vol. 63, no. 6, pp. 2056-2070, Jun. 2015.

[17] J. Zhang, O. Taghizadeh, and M. Haardt, "Transmit strategies for fullduplex point-to-point systems with residual self-interference," in Proc. Int. ITG Workshop on Smart Antennas (WSA 2013), 2013, pp. 1-8.

[18] O. Taghizadeh and R. Mathar, "Interference mitigation via power optimization schemes for full-duplex networking," in Proc. 19th Int. ITG Workshop on Smart Antennas (WSA), Mar. 2015, pp. 1-7.

[19] T. M. Kim, H. J. Yang, and A. Paulraj, "Distributed sum-rate optimization for full-duplex MIMO system under limited dynamic range," IEEE Signal Process. Letters, vol. 20, no. 6, pp. 555-558, Jun. 2013.

[20] D. Nguyen, L. Tran, P. Pirinen, and M. Latva-aho, "On the spectral efficiency of full-duplex small cell wireless systems," IEEE Trans. Wireless Commun., vol. 13, no. 9, pp. 4896-4910, Sep. 2014.

[21] S. Li, R. Murch, and V. Lau, "Linear transceiver design for full-duplex multi-user MIMO system," in IEEE Int. Conf. Commun. (ICC), Jun. 2014, pp. 4921-4926.

[22] Z. Ding, S. M. Perlaza, I. Esnaola, and H. V. Poor, "Power allocation strategies in energy harvesting wireless cooperative networks," IEEE Trans. Wireless Commun., vol. 13, no. 2, pp. 846-860, Feb. 2014.

[23] Z. Ding, C. Zhong, D. W. K. Ng, M. Peng, H. A. Suraweera, R. Schober, and H. V. Poor, "Application of smart antenna technologies in simultaneous wireless information and power transfer," IEEE Commun. Mag., vol. 53, pp. 86-93, Apr. 2015.

[24] G. Zhu, C. Zhong, H. A. Suraweera, G. K. Karagiannidis, Z. Zhang, and T. A. Tsiftsis, "Wireless information and power transfer in relay systems with multiple antennas and interference," IEEE Trans. Commun., vol. 63, pp. 1400-1418, Apr. 2015.

[25] A. A. Nasir, X. Zhou, S. Durrani, and R. Kennedy, "Relaying protocols for wireless energy harvesting and information processing," IEEE Trans. Wireless Commun., vol. 12, pp. 3622-3636, Jul. 2013.

[26] L. R. Varshney, "Transporting information and energy simultaneously," in IEEE Int. Symp. Inf. Theory (ISIT), no. 1612-1616, Toronto, Canada, Jul. 2008. 


$$
\begin{aligned}
& \left(\begin{array}{ll}
\xi_{i}^{(a)}\left(1-\rho_{i}^{(a)}\right) & \sqrt{\delta_{i}^{(a)}} \\
\sqrt{\delta_{i}^{(a)}} & V S 2+\bar{a}_{i i}^{a a}+\hat{\Sigma}_{i}^{(a)}
\end{array}\right) \succeq 0, \\
& \left(\begin{array}{cc}
\operatorname{vec}\left(\tilde{\mathbf{H}}_{i i}^{(a b)}\right)^{H}\left(\mathbf{W}_{i}^{b} \otimes \mathbf{I}\right) \operatorname{vec}\left(\tilde{\mathbf{H}}_{i i}^{(a b)}\right)-V S 2-\mu_{i i}^{a b} & \operatorname{vec}\left(\tilde{\mathbf{H}}_{i i}^{(a b)}\right)^{H}\left(\mathbf{W}_{i}^{b} \otimes \mathbf{I}\right) \\
\left(\operatorname{vec}\left(\tilde{\mathbf{H}}_{i i}^{(a b)}\right)^{H}\left(\mathbf{W}_{i}^{b} \otimes \mathbf{I}\right)\right)^{H} & \left(\mathbf{W}_{i}^{b} \otimes \mathbf{I}\right)+\frac{\mu_{i i}^{a b}}{\eta_{i i}^{a b 2}} \mathbf{I}
\end{array}\right) \succeq 0, \\
& \left(\begin{array}{cc}
\operatorname{vec}\left(\tilde{\mathbf{H}}_{i i}^{(a a)}\right)^{H}\left(\mathbf{W}_{i}^{a} \otimes \mathbf{I}\right) \operatorname{vec}\left(\tilde{\mathbf{H}}_{i i}^{(a a)}\right)-\bar{a}_{i i}^{a a}-\mu_{i i}^{a a} & \operatorname{vec}\left(\tilde{\mathbf{H}}_{i i}^{(a a)}\right)^{H}\left(\mathbf{W}_{i}^{a} \otimes \mathbf{I}\right) \\
\left(\operatorname{vec}\left(\tilde{\mathbf{H}}_{i i}^{(a a)}\right)^{H}\left(\mathbf{W}_{i}^{a} \otimes \mathbf{I}\right)\right)^{H} & \left(\mathbf{W}_{i}^{a} \otimes \mathbf{I}\right)+\frac{\mu_{i i}^{a a}}{\eta_{i i}^{a a}} \mathbf{I}
\end{array}\right) \succeq 0, \\
& \left(\begin{array}{cc}
\operatorname{vec}\left(\tilde{\mathbf{H}}_{i i}^{(a b)}\right)^{H}\left(\operatorname{diag}\left(\mathbf{W}_{i}^{b}\right) \otimes \mathbf{I}\right) \operatorname{vec}\left(\tilde{\mathbf{H}}_{i i}^{(a b)}\right)-\hat{a}_{i i}^{a b}-\mu_{i i}^{a b} & \operatorname{vec}\left(\tilde{\mathbf{H}}_{i i}^{(a b)}\right)^{H}\left(\operatorname{diag}\left(\mathbf{W}_{i}^{b}\right) \otimes \mathbf{I}\right) \\
\left(\operatorname{vec}\left(\tilde{\mathbf{H}}_{i i}^{(a b)}\right)^{H}\left(\operatorname{diag}\left(\mathbf{W}_{i}^{b}\right) \otimes \mathbf{I}\right)\right)^{H} & \left(\operatorname{diag}\left(\mathbf{W}_{i}^{b}\right) \otimes \mathbf{I}\right)+\frac{\mu_{i i}^{a b}}{\eta_{i i}^{a b 2}} \mathbf{I}
\end{array}\right) \\
& \succeq 0 \text {, } \\
& \left(\begin{array}{cc}
\operatorname{vec}\left(\tilde{\mathbf{H}}_{i i}^{(a a)}\right)^{H}\left(\operatorname{diag}\left(\mathbf{W}_{i}^{a}\right) \otimes \mathbf{I}\right) \operatorname{vec}\left(\tilde{\mathbf{H}}_{i i}^{(a a)}\right)-\hat{a}_{i i}^{a a}-\hat{\mu}_{i i}^{a a} & \operatorname{vec}\left(\tilde{\mathbf{H}}_{i i}^{(a a)}\right)^{H}\left(\operatorname{diag}\left(\mathbf{W}_{i}^{a}\right) \otimes \mathbf{I}\right) \\
\left(\operatorname{vec}\left(\tilde{\mathbf{H}}_{i i}^{(a a)}\right)^{H}\left(\operatorname{diag}\left(\mathbf{W}_{i}^{a}\right) \otimes \mathbf{I}\right)\right)^{H} & \left(\operatorname{diag}\left(\mathbf{W}_{i}^{a}\right) \otimes \mathbf{I}\right)+\frac{\hat{\mu}_{i i}^{a a}}{\eta_{i i}^{a a}} \mathbf{I}
\end{array}\right) \\
& \succeq 0 \text {, }
\end{aligned}
$$

[27] Q. Shi, W. Xu, L. Liu, and R. Zhang, "Joint transmit beamforming and receive power splitting for MISO swipt systems," IEEE Trans. Wireless Commun., vol. 13, no. 6, pp. 3269-3280, Jun. 2014.

[28] S. Biswas, S. Vuppala, and T. Ratnarajah, "On the performance of mmwave networks aided by wirelessly powered relays," IEEE Journal of Selected Topics in Signal Processing, vol. 10, no. 8, pp. 1522-1537, Dec 2016.

[29] A. H. Sakr and E. Hossain, "Analysis of $k$-tier uplink cellular networks with ambient RF energy harvesting," IEEE Journal on Selected Areas in Communications, vol. 33, no. 10, pp. 2226-2238, Oct 2015.

[30] G. Santella and F. Mazzenga, "A hybrid analytical-simulation procedure for performance evaluation in M-QAM-OFDM schemes in presence of nonlinear distortions," IEEE Trans. Veh. Technol., vol. 47, pp. 142-151, Feb. 1998.

[31] H. Suzuki, T. V. A. Tran, I. B. Collings, G. Daniels, and M. Hedley, "Transmitter noise effect on the performance of a MIMO-OFDM hardware implementation achieving improved coverage," IEEE J. Sel. Areas Commun., vol. 26, pp. 867-876, Aug. 2008.

[32] R. Zhang and C. Ho, "MIMO broadcasting for simultaneous wireless information and power transfer," IEEE Trans. Wireless Commun., vol. 12, no. 5, pp. 1989-2001, May 2013.

[33] N. D. Sidiropoulos, T. N. Davidson, and Z.-Q. Luo, "Transmit beamforming for physical-layer multicasting," IEEE Trans. Signal Process. vol. 54, no. 6, pp. 2239-2251, 2006.

[34] H. Yu and V. Lau, "Rank-constrained schur-convex optimization with multiple trace/log-det constraints," IEEE Trans. Signal Process., vol. 59, no. 1, pp. 304-314, 2011.

[35] K. Gomadam, V. Cadambe, and S. Jafar, "A distributed numerical approach to interference alignment and applications to wireless interfer- ence networks," IEEE Trans. Inf. Theory, vol. 57, no. 6, pp. 3309-3322, jun 2011.

[36] A. Ben-Tal and A. Nemirovski, "Lectures on modern convex optimization: Analysis, algorithms, engineering applications," 2001.

[37] R. Hunger, "Floating point operations in matrix-vector calculus," 2007.

[38] P. Ubaidulla and A. Chockalingam, "Relay precoder optimization in MIMO-relay networks with imperfect CSI," IEEE Trans. Signal Process., vol. 59, no. 11, pp. 5473-5484, Nov. 2011.

[39] S. Boyd and L. Vandenberghe, Convex Optimization. Cambridge, UK: Cambridge University Press, 2004.

[40] M. Grant and S. Boyd, "CVX: Matlab software for disciplined convex programming, version 2.1, March 2014.

[41] _ - "Graph implementations for non smooth convex programs, recent advances in learning and control (A tribute to M. Vidyasagar)," 2008.

[42] Z. Q. Luo, W. K. Ma, A. C. So, Y. Ye, and S. Zhang, "Semidefinite relaxation of quadratic optimization problems," IEEE Trans. Signal Process. Mag., vol. 27, no. 3, pp. 20-34, may 2010.

[43] N. S. K. Phan, S. Vorobyov and C. Tellambura, "Spectrum sharing in wireless networks via QoS-aware secondary multicast beamforming," IEEE Trans. Signal Process., vol. 57, no. 6, pp. 2323-2335, Jun 2009.

[44] A. C. Cirik, S. Biswas, S. Vuppala, and T. Ratnarajah, "Beamforming design for full-duplex MIMO interference channels: Qos and energy-efficiency considerations," IEEE Transactions on Communications, vol. 64, no. 11, pp. 4635-4651, Nov 2016.

[45] M. K. J. Jose, N. Prasad and S. Rangarajan, "On robust weightedsum rate maximization in MIMO interference networks," IEEE Int. Conf.Commun. (ICC), pp. 1-6, 2011. 


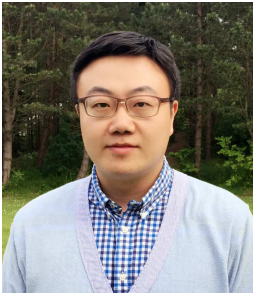

Jiang Xue (S'09-M'13) received the B.S. degree in Information and Computing Science from the Xi' an Jiaotong University, Xi' an, China, in 2005, the M.S. degrees in Applied Mathematics from Lanzhou University, China and Uppsala University, Sweden, in 2008 and 2009, respectively. Dr. J. Xue received the $\mathrm{Ph} . \mathrm{D}$. degree in Electrical and Electronic Engineering from ECIT, the Queen's University of Belfast, U.K., in 2012. From 2013 to 2017, He was a Research Fellow with the University of Edinburgh, U.K..

Since 2017, Dr. J. Xue has joined the National Engineering Laboratory for Big Data Analytics, Xi'an International Academy for Mathematics and Mathematical Technology, School of Mathematics and Statistics in Xi'an Jiaotong University, China, as a professor. His main interests include the machine learning and wireless communication, performance analysis of general multiple antenna systems, stochastic geometry, cooperative communications, and cognitive radio.

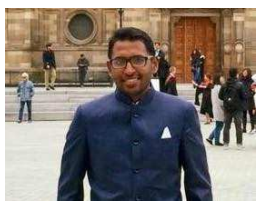

Sudip Biswas (''16-M'17) received the B.Tech. degree in electronics and communication engineering from the Sikkim Manipal Institute of Technology, Sikkim, India, in 2010, the M.Sc. degree in signal processing and communications from the University of Edinburgh, Edinburgh, U.K., in 2013 and the Ph.D. degree in digital communications at the University of Edinburgh?s Institute for Digital Communications in 2017. Currently, he is working as a research scientist at the institute for Digital Communications, University of Edinburgh. His research interests include various topics in wireless communications and network information theory with particular focus possible 5G technologies such as massive MIMO, mmWave, full-duplex, NOMA and wireless caching.

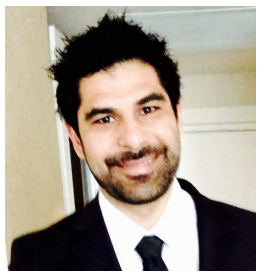

Ali Cagatay Cirik (S13-M14) received the B.S and M.S. degrees in telecommunications and electronics engineering from Sabanci University, Istanbul, Turkey, in 2007 and 2009, respectively, and $\mathrm{Ph} . \mathrm{D}$. degree in electrical engineering from University of California, Riverside in 2014. He held research fellow positions at Centre for Wireless Communications, Oulu, Finland, University of Edinburgh, U.K and University of British Columbia, Vancouver, Canada between June 2014 and October 2017. His industry experience includes internships at Mitsubishi Electric Research Labs (MERL), Cambridge, MA, in 2012, Broadcom Corporation, Irvine, CA, in 2013 and includes industrial postdoctoral researcher position at Sierra Wireless, Richmond, Canada between November 2015 and October 2017. He is currently working at Ofinno Technologies, Herndon, VA as Senior Technical Staff. His primary research interests are fullduplex communication, 5G non-orthogonal multiple-access (NOMA), MIMO signal processing, and convex optimization.

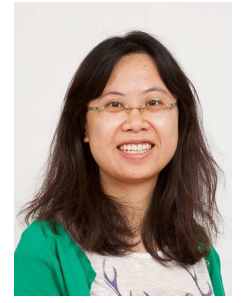

Huiqin Du received the B.Sc. in Electronic Information Science and Technical from Beijing University of Chemical Technology, China in 2004, MSc. with distinction in Radio Frequency Communication System in 2006 and PhD degree in Signal Processing in 2010, from University of Southampton and University of Edinburgh, respectively. From 20102013, She worked as a research fellow at Queen's University of Belfast and University of Edinburgh. Since 2013, she has been with the College of Information Science and Technology, Jinan University, Guangzhou, China as an Associate Professor. Her current research interests include statistical signal processing, wireless MIMO communications, cognitive radio network, massive MIMO and hybrid beamforming.

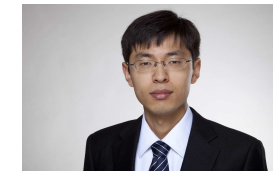

Yang Yang received the B.S. degree in School of Information Science and Engineering, Southeast University, Nanjing, China, in 2009, and the Ph.D. degree in Department of Electronic and Computer Engineering, The Hong Kong University of Science and Technology. From Nov. 2013 to Nov. 2015 he had been a postdoctoral research associate at the Communication Systems Group, Darmstadt University of Technology, Darmstadt, Germany. From Dec. 2015 to Oct. 2017 he had been a senior standards and research scientist in Intel, Germany. He joined the University of Luxembourg as a research associate in Nov. 2017. His research interests are in parallel and distributed solution methods in convex optimization and nonlinear programming, with applications in communication networks and signal processing.

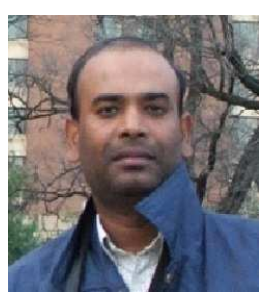

Tharmalingam Ratnarajah (A'96-M'05-SM'05) is currently with the Institute for Digital Communications, University of Edinburgh, Edinburgh, UK, as a Professor in Digital Communications and Signal Processing and the Head of Institute for Digital Communications. His research interests include signal processing and information theoretic aspects of 5G and beyond wireless networks, full-duplex radio, mmWave communications, random matrices theory, interference alignment, statistical and array signal processing and quantum information theory. He has published over 330 publications in these areas and holds four U.S. patents. He was the coordinator of the FP7 projects ADEL (3.7M Euro) in the area of licensed shared access for $5 \mathrm{G}$ wireless networks and HARP (3.2M Euro) in the area of highly distributed MIMO and FP7 Future and Emerging Technologies projects HIATUS (2.7M Euro) in the area of interference alignment and CROWN (2.3M Euro) in the area of cognitive radio networks. Dr Ratnarajah is a Fellow of Higher Education Academy (FHEA), U.K.

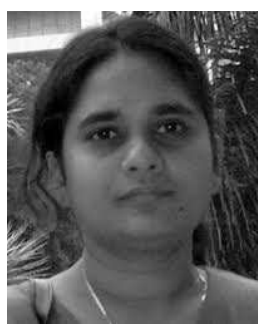

Mathini Sellathurai (S95M02SM06) is a Full Professor of signal processing and intelligent systems with Heriot-Watt University, Edinburgh, U.K. In her 15-year research on Signal Processing for Communications, she has made seminal contributions on MIMO wireless systems. She has published 200 IEEE entries with over 2400 citations, given invited talks and has written a book and several book chapters in topics related to this project. She was a recipient of the IEEE Communication Society Fred W. Ellersick Best Paper Award in 2005, the Industry Canada Public Service Awards for contributions in science and technology in 2005, and the Best Ph.D. Thesis Award (Silver Medal) from NSERC Canada in 2002. She is also a member for IEEE SPCOM Technical Strategy Committee, an Editor of IEEE TSP from 2009. She is also the General CoChair of IEEE SPAWC2016 in Edinburgh. She is a fellow of Higher Education Academy, U.K. 